\title{
Verifying Multi-threaded Software using SMT-based Context-Bounded Model Checking
}

\section{Lucas Cordeiro and Bernd Fischer lucascordeiro@ufam.edu.br}

\author{
Southâmpton \\ School of Electronics \\ and Computer Science
}

UFAM 


\section{Bounded Model Checking (BMC)}

Basic Idea: check negation of given property up to given depth

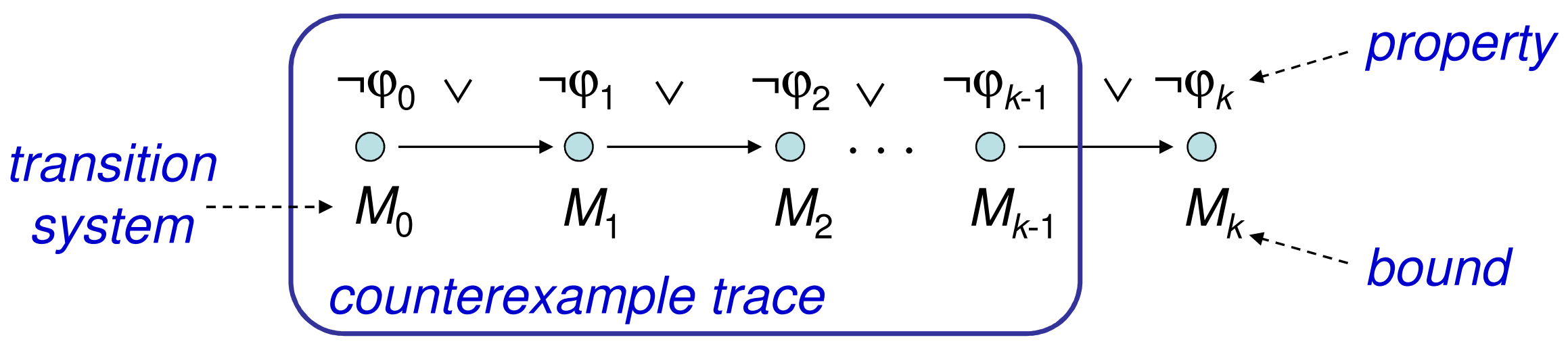

- transition system M unrolled $k$ times

- for programs: unroll loops, unfold arrays, ...

- translated into verification condition $\psi$ such that $\psi$ satisfiable iff $\varphi$ has counterexample of max. depth $k$

- has been applied successfully to verify (embedded) software 


\section{BMC of Multi-threaded Software}

- concurrency bugs are tricky to reproduce/debug because they usually occur under specific thread interleavings

- most common errors: 67\% related to atomicity and order violations, 30\% related to deadlock [Lu et al.'08]

- problem: the number of interleavings grows exponentially with the number of threads and program statements

- context switches among threads increase the number of possible executions

- two important observations help us:

- concurrency bugs are shallow [Qadeer\&Rehof'05]

- SAT/SMT solvers produce unsatisfiable cores that allow us to remove logic that is not relevant 


\section{Objective of this work}

\section{Exploit SMT to improve BMC of multi-threaded software}

- exploit SMT solvers to:

- prune the property and data dependent search space (nonchronological backtracking and conflict clauses learning)

- remove interleavings that are not relevant by analyzing the proof of unsatisfiability

- propose three approaches to SMT-based BMC:

- lazy exploration of the interleavings

- schedule guards to encode all interleavings

- underapproximation and widening (UW) [Grumberg\&et al.'05]

- implement these approaches in ESBMC and evaluate them using multi-threaded applications 


\section{Lazy exploration of interleavings}

Idea: iteratively generate all possible interleavings and call the BMC procedure on each interleaving

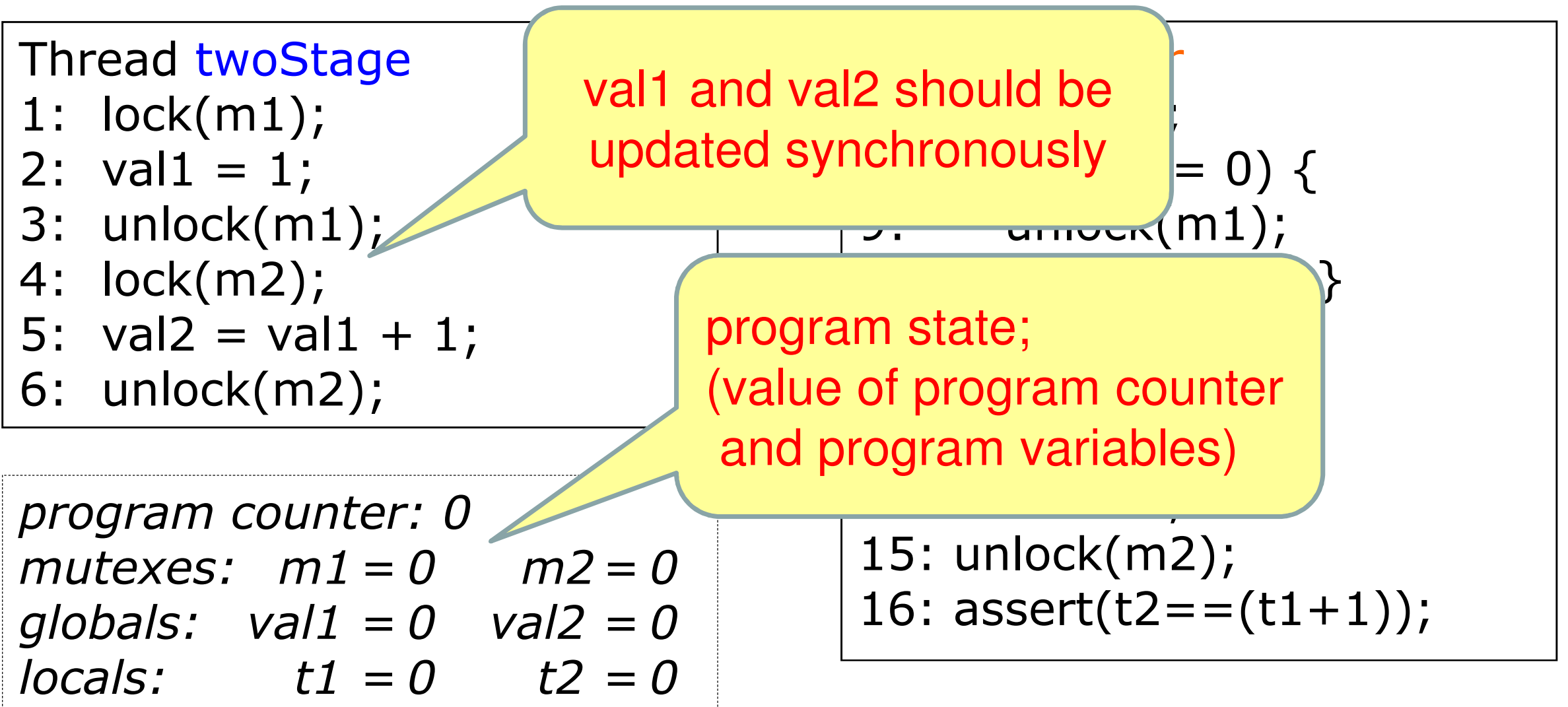




\section{Lazy exploration of interleavings}

Idea: iteratively generate all possible interleavings and call the BMC procedure on each interleaving

interleaving \#1: 1

\begin{tabular}{|c|}
\hline Thread twoStage \\
\hline $\begin{array}{l}\text { 1: } \operatorname{lock}(\mathbf{m} 1) ; \\
\text { 2: }\end{array}$ \\
\hline $\begin{array}{l}\text { 2: val1 }=1 ; \\
\text { 3: unlock }(m 1)\end{array}$ \\
\hline 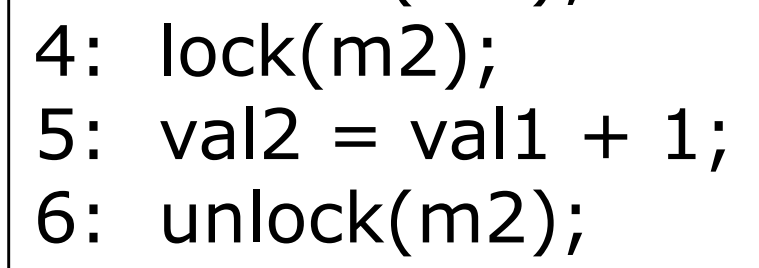 \\
\hline
\end{tabular}

program counter: 1 mutexes: $\mathbf{m 1}=\mathbf{1} \quad m 2=0$ globals: $v a l 1=0$ val $=0$ locals: $\quad t 1=0 \quad t 2=0$

Thread reader
7: $\operatorname{lock}(\mathrm{m} 1) ;$
8: if $(\operatorname{val} 1==0)\{$
9: $\operatorname{unlock}(\mathrm{m} 1) ;$
10: return NULL; $\}$
11: $\mathrm{t} 1=\operatorname{val} 1 ;$
12: unlock $(\mathrm{m} 1) ;$
13: lock $(\mathrm{m} 2) ;$
14: $\mathrm{t} 2=\mathrm{val} 2 ;$
15: unlock $(\mathrm{m} 2) ;$
16: $\operatorname{assert}(\mathrm{t} 2==(\mathrm{t} 1+1)) ;$




\section{Lazy exploration of interleavings}

Idea: iteratively generate all possible interleavings and call the BMC procedure on each interleaving

interleaving \#1: 1-2

\begin{tabular}{|c|}
\hline $\begin{array}{l}\text { Thread twoStage } \\
\text { 1: } \operatorname{lock}(\mathrm{m} 1)\end{array}$ \\
\hline 2: val1 = \\
\hline $\begin{array}{l}\text { 3: } \operatorname{unlock}(\mathrm{m} 1) \\
\text { 4: } \operatorname{lock}(\mathrm{m} 2) ;\end{array}$ \\
\hline $\begin{array}{l}\text { 4: } \operatorname{lock}(\mathrm{m} 2) \\
5: \quad \text { val2 }=\text { val1 }+1\end{array}$ \\
\hline $\begin{array}{l}\text { 5: } \text { val2 }=\text { val1 }+1 ; \\
6: \text { unlock }(m 2) ;\end{array}$ \\
\hline
\end{tabular}

program counter: 2 mutexes: $m 1=1 \quad m 2=0$ globals: $v a l 1=1$ val $=0$ locals: $\quad t 1=0 \quad t 2=0$

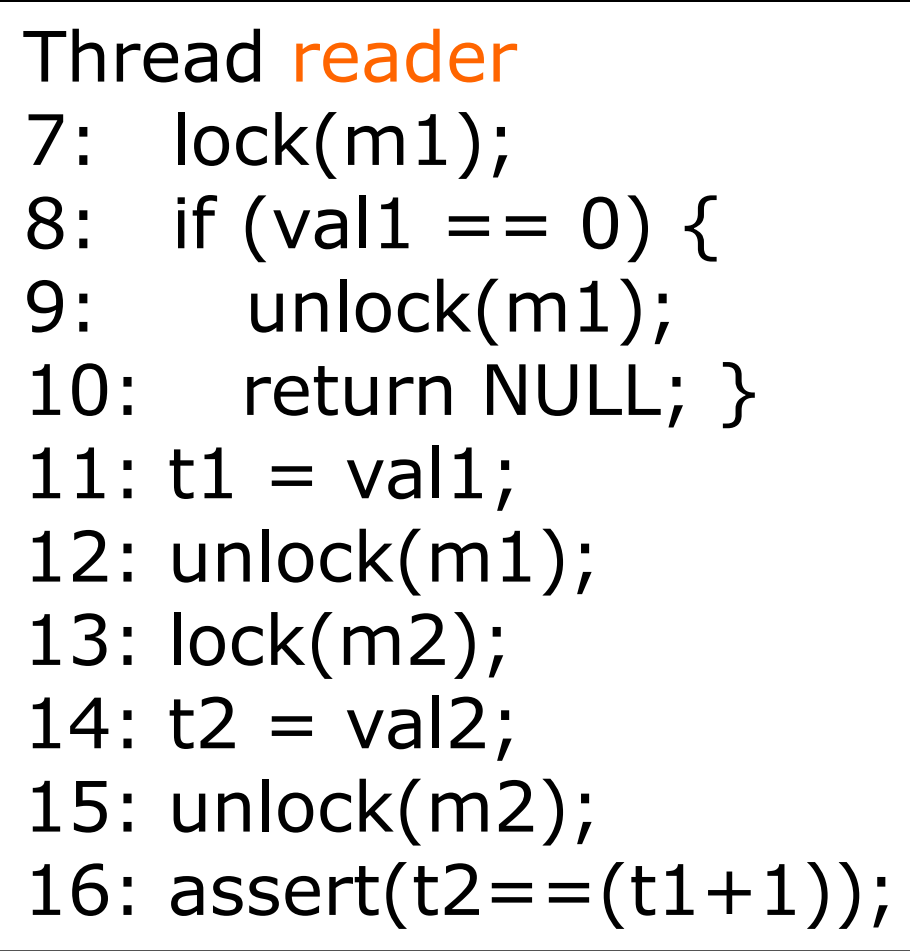




\section{Lazy exploration of interleavings}

Idea: iteratively generate all possible interleavings and call the BMC procedure on each interleaving

interleaving \#1: 1-2-3

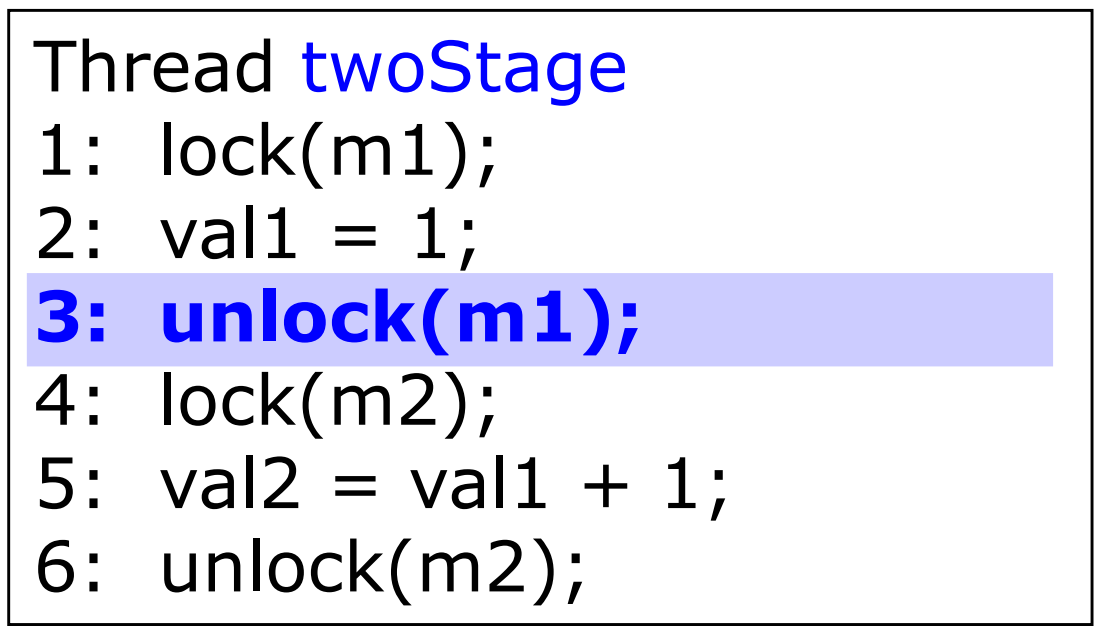

program counter: 3 mutexes: $\mathbf{m 1}=\mathbf{0} \quad m 2=0$ globals: $v a l 1=1 \quad$ val $2=0$ locals: $\quad t 1=0 \quad t 2=0$

Thread reader
7: $\operatorname{lock}(\mathrm{m} 1) ;$
8: if $(\operatorname{val} 1==0)\{$
9: $\operatorname{unlock}(\mathrm{m} 1) ;$
10: return NULL; $\}$
11: $\mathrm{t} 1=\operatorname{val} 1 ;$
12: unlock $(\mathrm{m} 1) ;$
13: lock $(\mathrm{m} 2) ;$
14: $\mathrm{t} 2=\mathrm{val} 2 ;$
15: unlock $(\mathrm{m} 2) ;$
16: $\operatorname{assert}(\mathrm{t} 2==(\mathrm{t} 1+1)) ;$




\section{Lazy exploration of interleavings}

Idea: iteratively generate all possible interleavings and call the BMC procedure on each interleaving

interleaving \#1: 1-2-3-7

Thread twoStage
1: lock $(\mathrm{m} 1) ;$
2: val1 $=1 ;$
3: unlock $(\mathrm{m} 1) ;$
4: lock $(\mathrm{m} 2) ;$
5: val2 $=$ val1 $1+1 ;$
6: unlock(m2);
program counter: 7
mutexes: $\mathbf{m 1}=\mathbf{1} \quad \mathrm{m} 2=0$
globals: val1 $=1 \quad$ val2 $=0$
locals: $\quad t 1=0 \quad$ t2 $=0$

Thread reader

8: $\quad$ if $($ val $1==0)\{$

9: $\quad$ unlock $(\mathrm{m} 1)$;

10: return $\mathrm{NULL} ;\}$

11: $\mathrm{t} 1$ = val1

12: unlock $(\mathrm{m} 1)$;

13: $\operatorname{lock}(\mathrm{m} 2)$;

14: $\mathrm{t} 2$ = val2;

15: $\operatorname{unlock}(\mathrm{m} 2)$;

16: $\operatorname{assert}(\mathrm{t} 2==(\mathrm{t} 1+1))$; 


\section{Lazy exploration of interleavings}

Idea: iteratively generate all possible interleavings and call the BMC procedure on each interleaving

interleaving \#1: 1-2-3-7-8

\begin{tabular}{|c|c|}
\hline 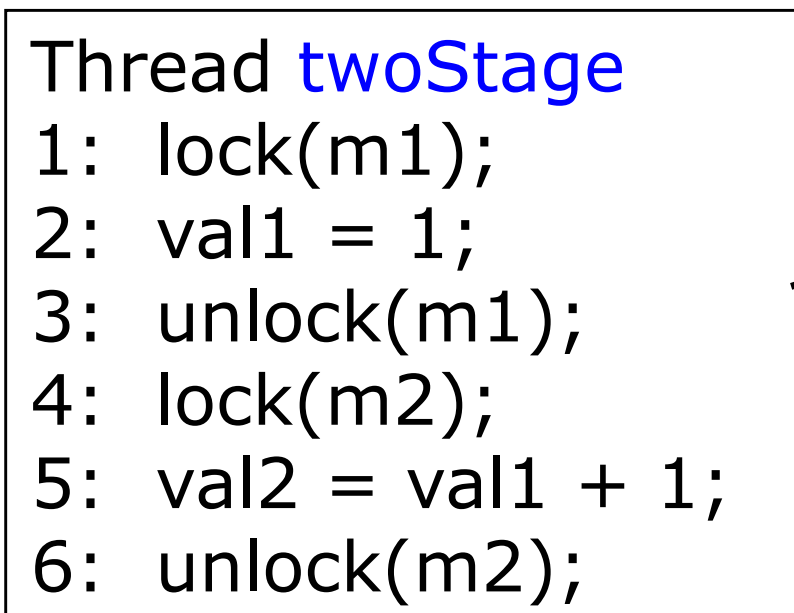 & \\
\hline
\end{tabular}

program counter: 8 mutexes: $m 1=1 \quad m 2=0$ globals: $v a l 1=1 \quad$ val $2=0$ locals: $\quad t 1=0 \quad t 2=0$
Thread reader

7: $\operatorname{lock}(\mathrm{m} 1)$;

8: if $(\operatorname{val} 1==0)$

10: return NULL; $\}$

11: $\mathrm{t} 1$ = val1;

12: $\operatorname{unlock}(\mathrm{m} 1)$;

13: $\operatorname{lock}(\mathrm{m} 2)$;

14: $\mathrm{t} 2$ = val2;

15: unlock $(\mathrm{m} 2)$;

16: assert $(\mathrm{t} 2==(\mathrm{t} 1+1))$; 


\section{Lazy exploration of interleavings}

Idea: iteratively generate all possible interleavings and call the BMC procedure on each interleaving

interleaving \#1: 1-2-3-7-8-11

Thread twoStage
1: lock $(\mathrm{m} 1) ;$
2: val1 $=1 ;$
3: unlock $(\mathrm{m} 1) ;$
4: lock $(\mathrm{m} 2) ;$
5: $\operatorname{val} 2=\mathrm{val} 1+1 ;$
6: unlock $(\mathrm{m} 2) ;$

program counter: 11 mutexes: $m 1=1 \quad m 2=0$ globals: val $1=1$ val $=0$ locals: $\quad t 1=1 \quad t 2=0$
Thread reader

7: $\operatorname{lock}(\mathrm{m} 1)$;

8: if $($ val $1==0)\{$

9: $\quad$ unlock $(\mathrm{m} 1)$;

10: return NULL; $\}$

12: $\operatorname{unlock}(\mathrm{m} 1)$;

13: $\operatorname{lock}(\mathrm{m} 2)$;

14: $\mathrm{t} 2$ = val2;

15: unlock $(\mathrm{m} 2)$;

16: $\operatorname{assert}(\mathrm{t} 2==(\mathrm{t} 1+1))$; 


\section{Lazy exploration of interleavings}

Idea: iteratively generate all possible interleavings and call the BMC procedure on each interleaving

interleaving \#1: 1-2-3-7-8-11-12

\begin{tabular}{|c|c|}
\hline 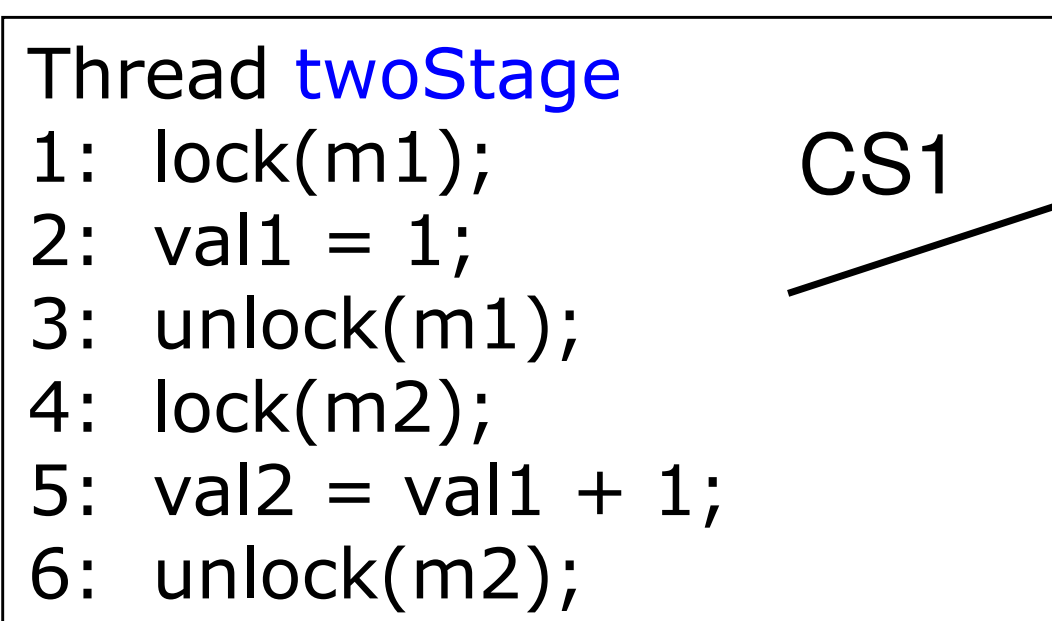 & $\begin{array}{l}\text { Thread reader } \\
\text { 7: lock }(\mathrm{m} 1) ; \\
\text { 8: if }(\operatorname{val} 1=0)\{ \\
9: \quad \text { unlock }(\mathrm{m} 1) ; \\
\text { 10: return } \mathrm{NULL} ;\} \\
\text { 11: } \mathrm{t} 1=\operatorname{val} 1 ; \\
12: \text { unlock }(\mathrm{m} 1) ;\end{array}$ \\
\hline $\begin{array}{l}\text { program counter: } 12 \\
\text { mutexes: } \boldsymbol{m} \mathbf{1}=\mathbf{0} \quad \mathrm{m} 2=0 \\
\text { globals: val1 }=1 \quad \text { val2 }=0\end{array}$ & $\begin{array}{l}\text { 13: } \operatorname{lock}(\mathrm{m} 2) ; \\
\text { 14: }=\mathrm{val} 2 ; \\
\text { 15: unlock }(\mathrm{m} 2) ; \\
\text { 16: } \operatorname{assert}(\mathrm{t} 2==(\mathrm{t} 1+1)) ;\end{array}$ \\
\hline
\end{tabular}

locals: $\quad t 1=1 \quad t 2=0$




\section{Lazy exploration of interleavings}

Idea: iteratively generate all possible interleavings and call the BMC procedure on each interleaving

interleaving \#1: 1-2-3-7-8-11-12-4

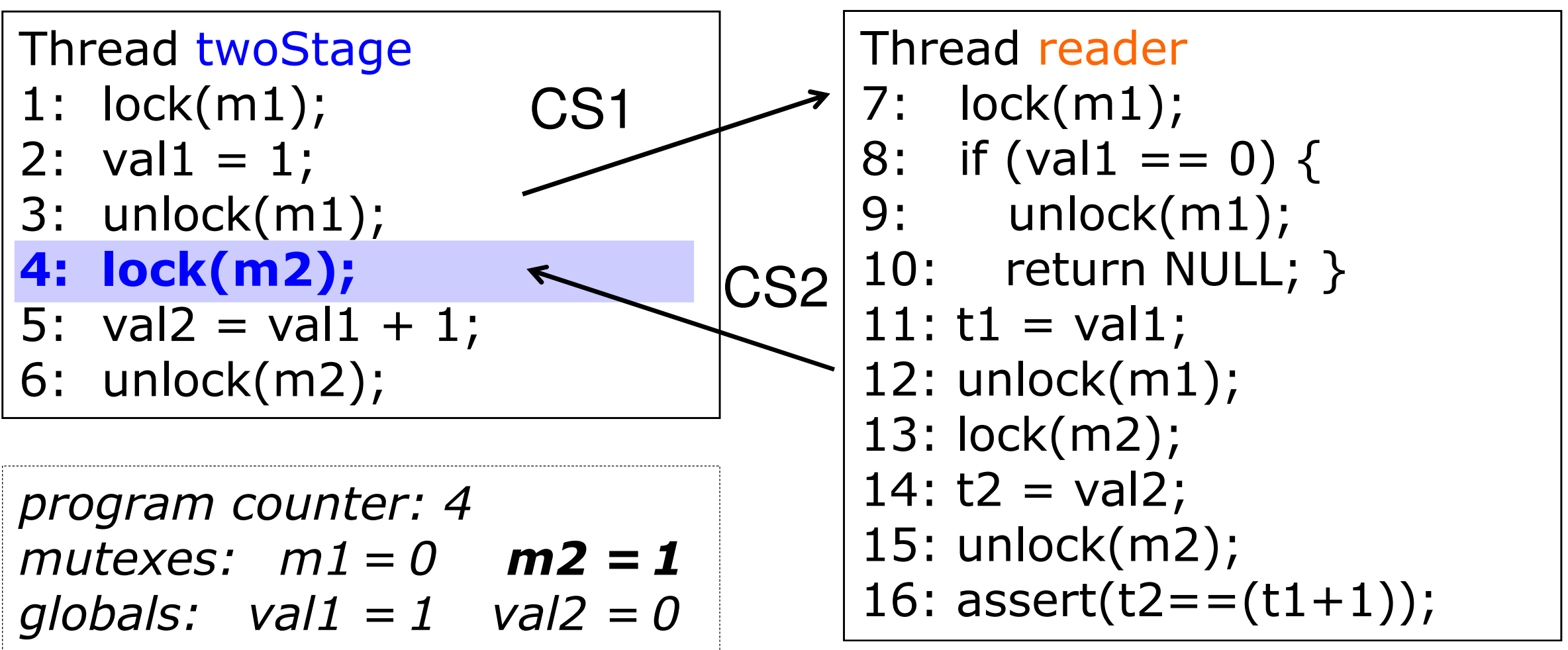

locals: $\quad t 1=1 \quad t 2=0$




\section{Lazy exploration of interleavings}

Idea: iteratively generate all possible interleavings and call the BMC procedure on each interleaving

interleaving \#1: 1-2-3-7-8-11-12-4-5

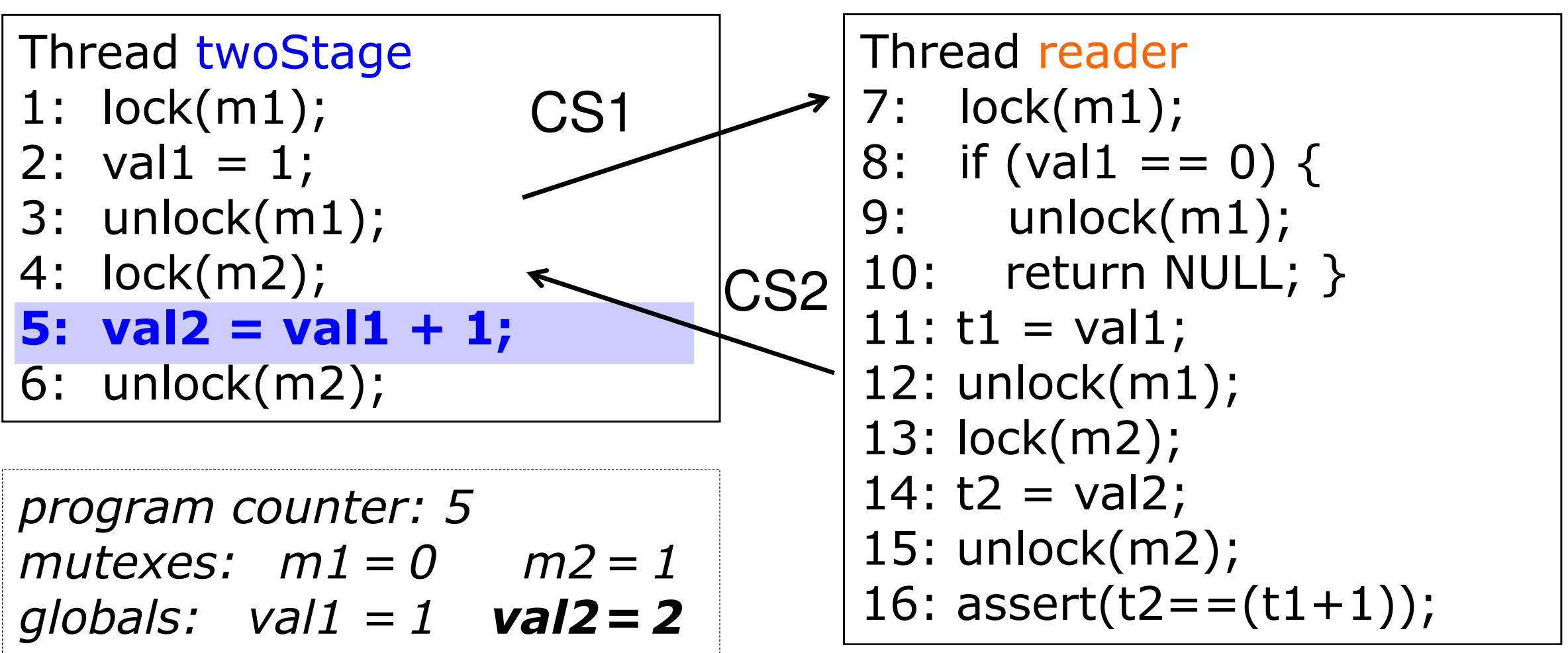

locals: $\quad t 1=1 \quad t 2=0$




\section{Lazy exploration of interleavings}

Idea: iteratively generate all possible interleavings and call the BMC procedure on each interleaving

interleaving \#1: 1-2-3-7-8-11-12-4-5-6

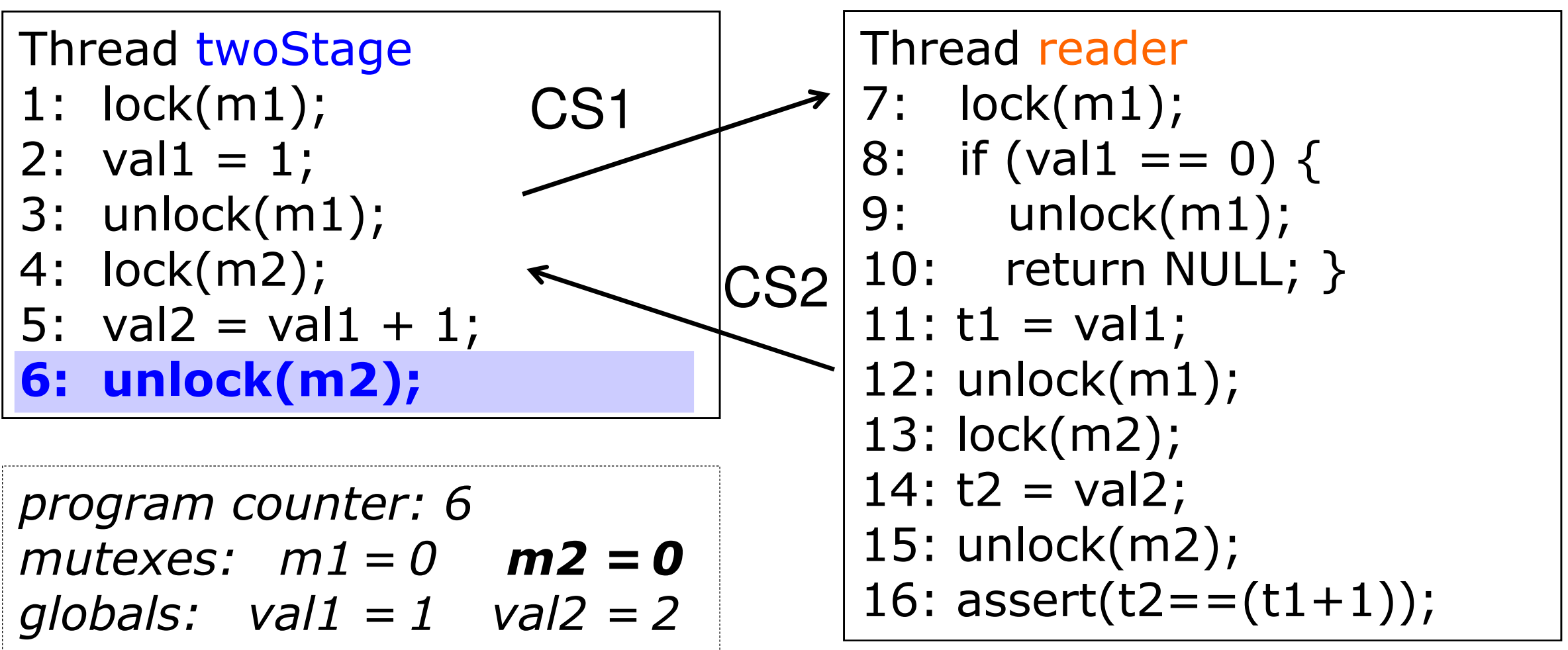

locals: $\quad t 1=1 \quad t 2=0$




\section{Lazy exploration of interleavings}

Idea: iteratively generate all possible interleavings and call the BMC procedure on each interleaving

interleaving \#1: 1-2-3-7-8-11-12-4-5-6-13

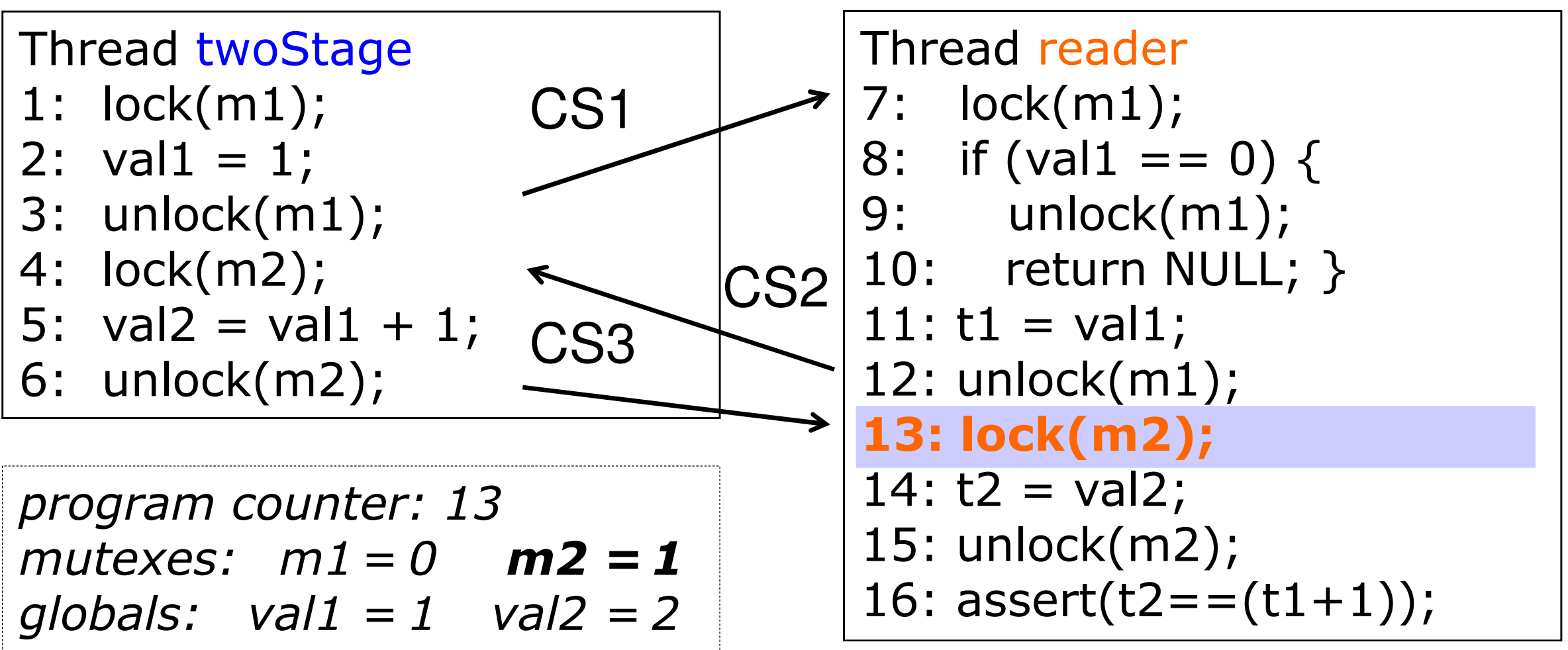

locals: $\quad t 1=1 \quad t 2=0$




\section{Lazy exploration of interleavings}

Idea: iteratively generate all possible interleavings and call the BMC procedure on each interleaving

interleaving \#1: 1-2-3-7-8-11-12-4-5-6-13-14

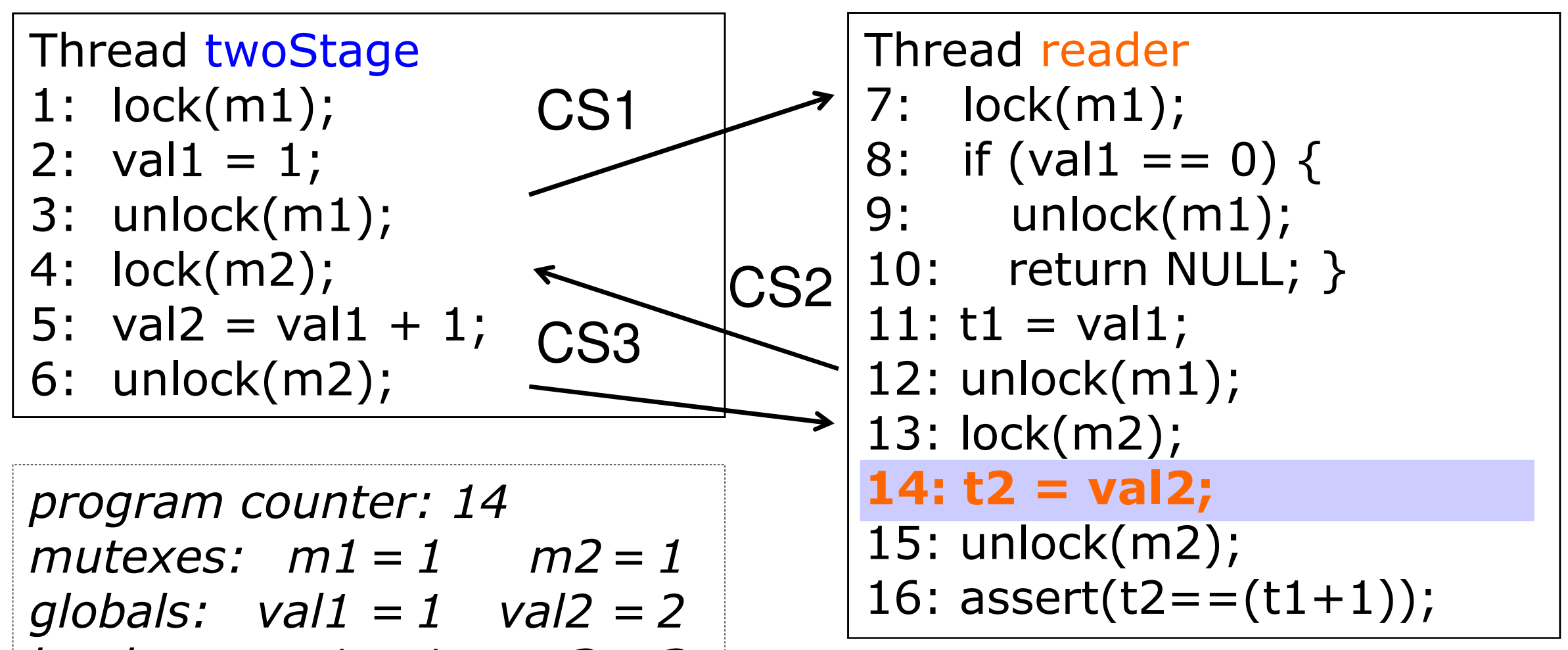
locals: $\quad t 1=1 \quad t 2=2$ 


\section{Lazy exploration of interleavings}

Idea: iteratively generate all possible interleavings and call the BMC procedure on each interleaving

interleaving \#1: 1-2-3-7-8-11-12-4-5-6-13-14-15

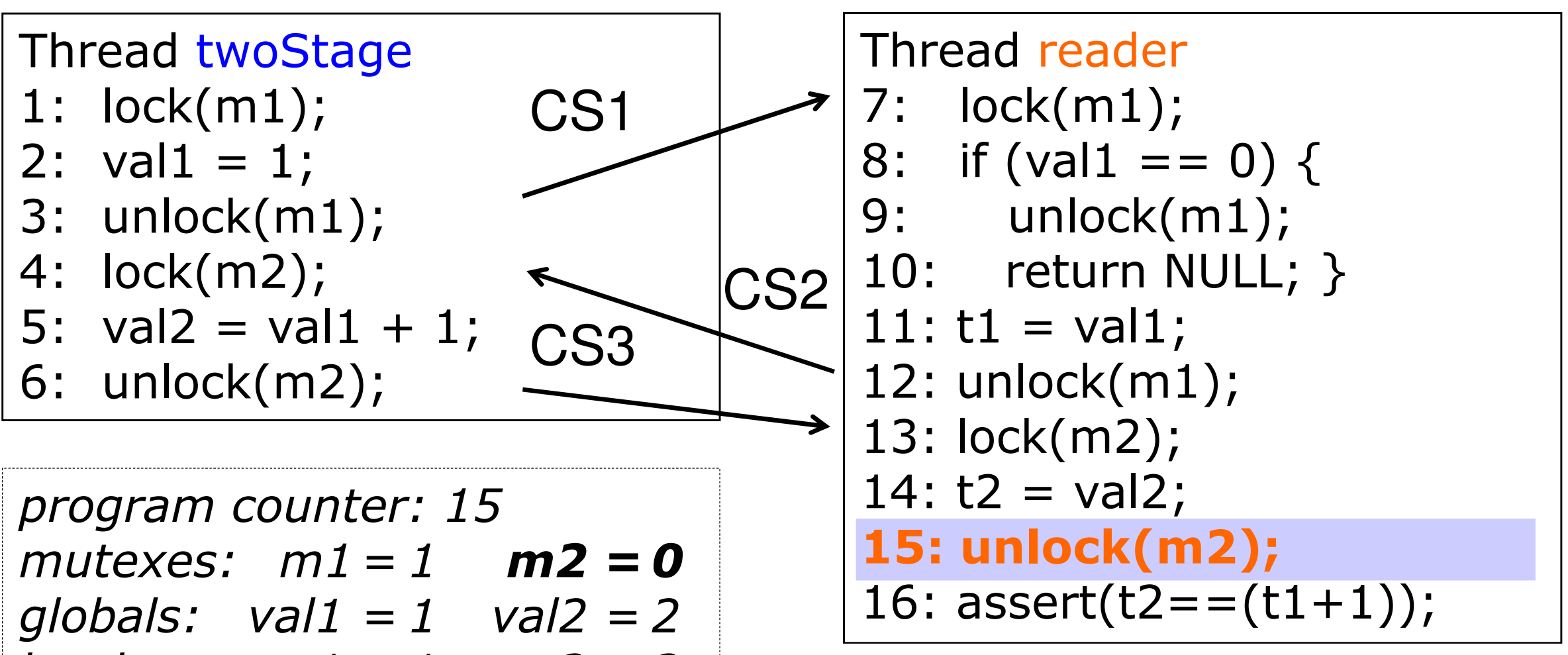

locals: $\quad t 1=1 \quad t 2=2$




\section{Lazy exploration of interleavings}

Idea: iteratively generate all possible interleavings and call the BMC procedure on each interleaving

interleaving \#1: 1-2-3-7-8-11-12-4-5-6-13-14-15-16

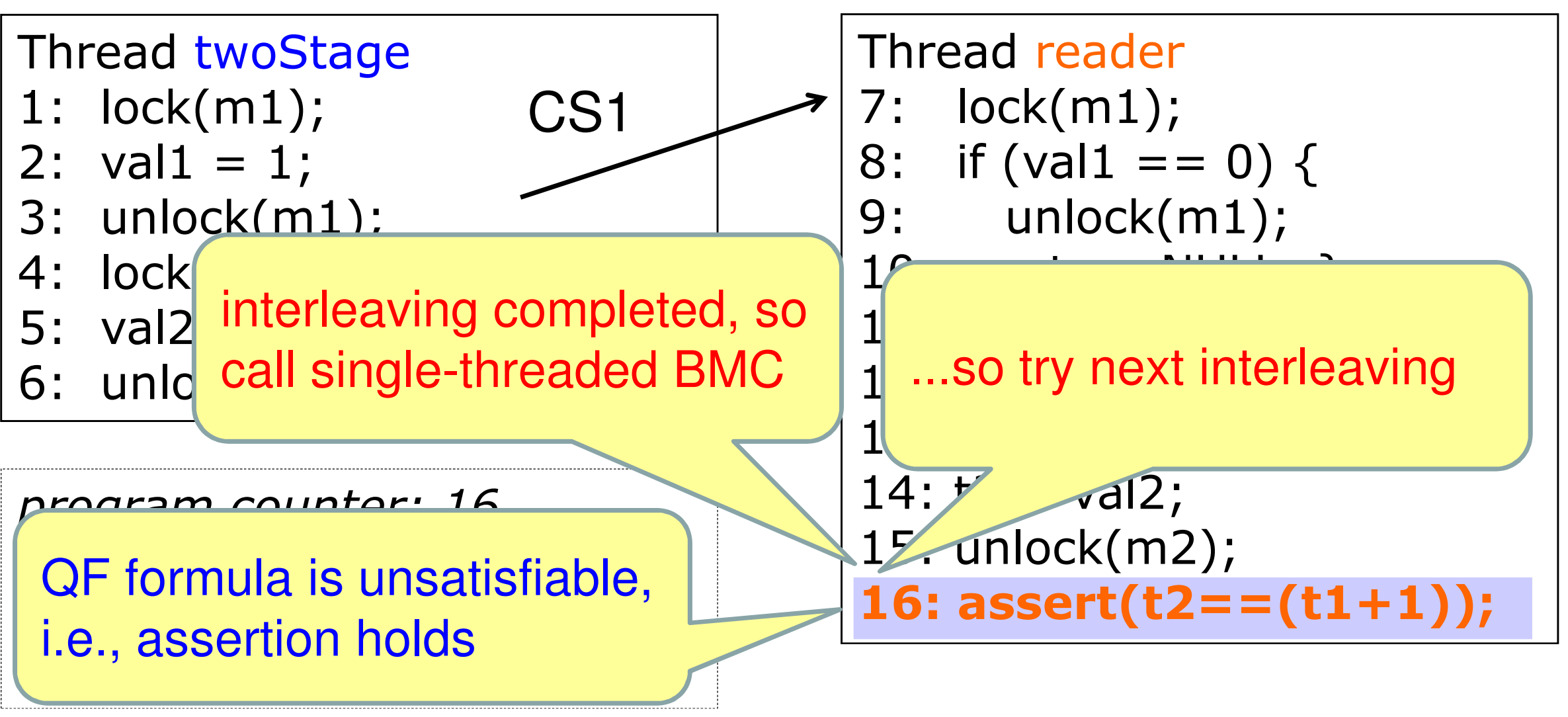




\section{Lazy exploration of interleavings}

Idea: iteratively generate all possible interleavings and call the BMC procedure on each interleaving

interleaving \#2:

Thread twoStage

1: $\operatorname{lock}(\mathrm{m} 1)$;

2: val1 = 1;

3: $\operatorname{unlock}(\mathrm{m} 1)$;

4: $\operatorname{lock}(\mathrm{m} 2)$;

5: val2 $=$ val1 +1 ;

6: unlock $(\mathrm{m} 2)$;

program counter: 0 mutexes: $m 1=0 \quad m 2=0$ globals: $v a l 1=0 \quad$ val $2=0$ locals: $\quad t 1=0 \quad t 2=0$
Thread reader
7: $\operatorname{lock}(\mathrm{m} 1)$;
8: if $($ val1 $==0)\{$
9: $\quad$ unlock $(\mathrm{m} 1)$;
10: return NULL; $\}$
11: $\mathrm{t} 1$ = val1;
12: unlock $(\mathrm{m} 1)$;
13: $\operatorname{lock}(\mathrm{m} 2)$;
14: t2 = val2;
15: unlock $(\mathrm{m} 2)$;
16: assert $(\mathrm{t} 2==(\mathrm{t} 1+1))$; 


\section{Lazy exploration of interleavings}

Idea: iteratively generate all possible interleavings and call the BMC procedure on each interleaving

interleaving \#2: 1-2-3

\begin{tabular}{|c|}
\hline 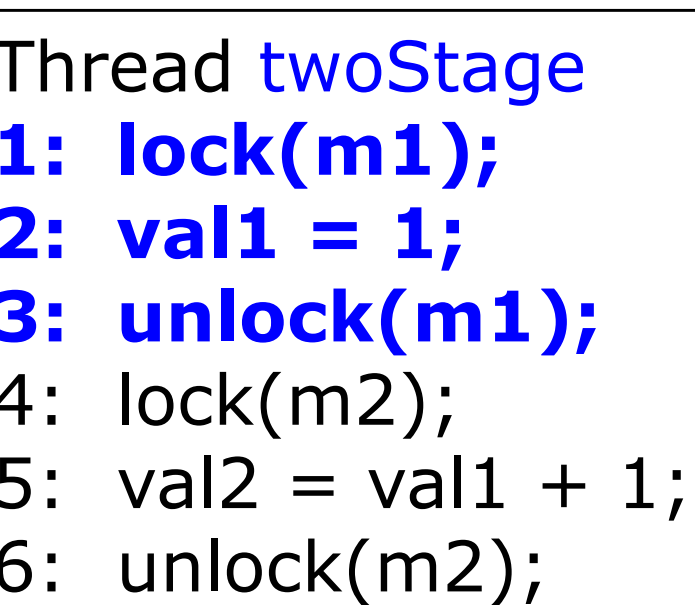 \\
\hline
\end{tabular}

program counter: 3 mutexes: $m 1=0 \quad m 2=0$ globals: $v a l 1=1 \quad$ val $2=0$ locals: $\quad t 1=0 \quad t 2=0$

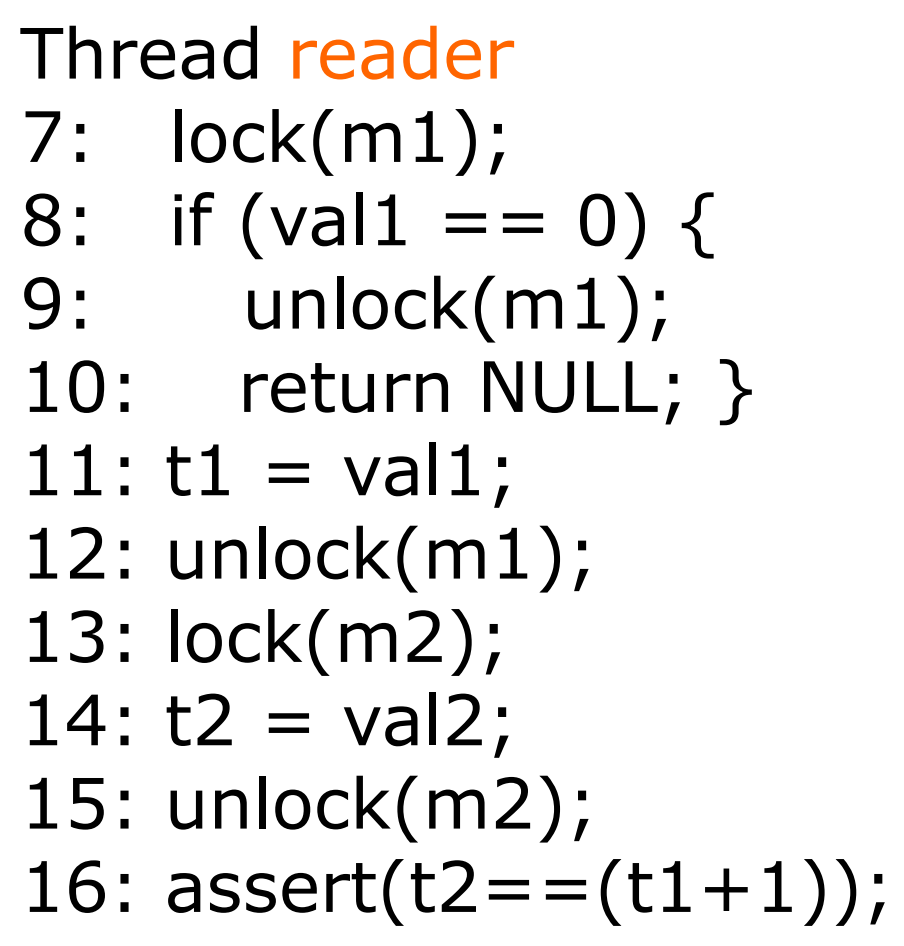




\section{Lazy exploration of interleavings}

Idea: iteratively generate all possible interleavings and call the BMC procedure on each interleaving

interleaving \#2: 1-2-3-7

Thread twoStage
1: lock $(\mathrm{m} 1) ;$
2: val1 $=1 ;$
3: unlock $(\mathrm{m} 1) ;$
4: lock $(\mathrm{m} 2) ;$
5: val2 $=$ val1 $1+1 ;$
6: unlock(m2);
program counter: 7
mutexes: $\mathbf{m 1}=\mathbf{1} \quad \mathrm{m} 2=0$
globals: val1 $=1 \quad$ val2 $=0$
locals: $\quad t 1=0 \quad$ t2 $=0$

Thread reader

8: if $($ val $1==0)\{$

9: $\quad$ unlock $(\mathrm{m} 1)$;

10: return $\mathrm{NULL} ;\}$

11: $\mathrm{t} 1$ = val1

12: unlock (m1);

13: $\operatorname{lock}(\mathrm{m} 2)$;

14: $\mathrm{t} 2$ = val2;

15: $\operatorname{unlock}(\mathrm{m} 2)$;

16: $\operatorname{assert}(\mathrm{t} 2==(\mathrm{t} 1+1))$; 


\section{Lazy exploration of interleavings}

Idea: iteratively generate all possible interleavings and call the BMC procedure on each interleaving

interleaving \#2: 1-2-3-7-8-11-12-13-14-15-16

\begin{tabular}{|c|c|}
\hline \multicolumn{2}{|l|}{ Thread tw } \\
\hline 1: $\operatorname{lock}(\mathrm{m} 1)$; & 7: $\operatorname{lock}(\mathrm{m} 1)$; \\
\hline 2: val1 $=1 ;$ & 8: if $(\mathrm{val1}==0)$ \\
\hline 3: unlock $(\mathrm{m} 1)$; & 9: $\quad$ unlock $(\mathrm{m} 1)$ \\
\hline $\begin{array}{l}\text { 4: } \operatorname{lock}(\mathrm{m} 2) ; \\
\text { 5: } \text { val }=\text { val } 1+1:\end{array}$ & 10: return $N U L L ;\}$ \\
\hline 6: unlock $(\mathrm{m} 2)$ & 12: unlock (m1): \\
\hline & $\begin{array}{l}\text { 13: lock(m2); } \\
14: \text { t2 = val2; }\end{array}$ \\
\hline $\begin{array}{l}\text { mutexes: } m 1=0 \quad m 2=0 \\
\text { globals: val }=1 \text { val2 }=0\end{array}$ & $\begin{array}{l}\text { 15: unlock }(\mathrm{m} 2) ; \\
16: \operatorname{assert}(\mathrm{t} 2==(\mathrm{t} 1+1))\end{array}$ \\
\hline
\end{tabular}




\section{Lazy exploration of interleavings}

Idea: iteratively generate all possible interleavings and call the BMC procedure on each interleaving

interleaving \#2: 1-2-3-7-8-11-12-13-14-15-16-4

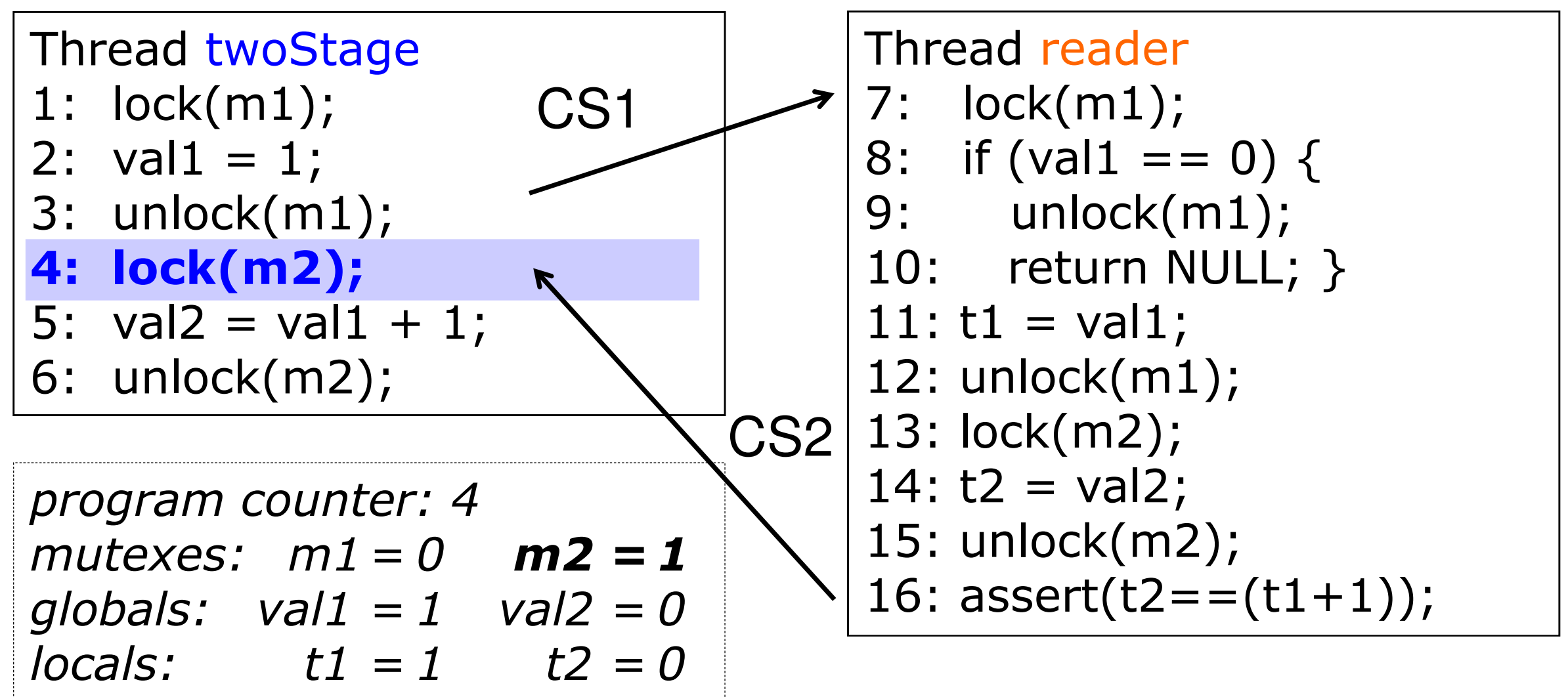




\section{Lazy exploration of interleavings}

Idea: iteratively generate all possible interleavings and call the BMC procedure on each interleaving

interleaving \#2: 1-2-3-7-8-11-12-13-14-15-16-4-5-6

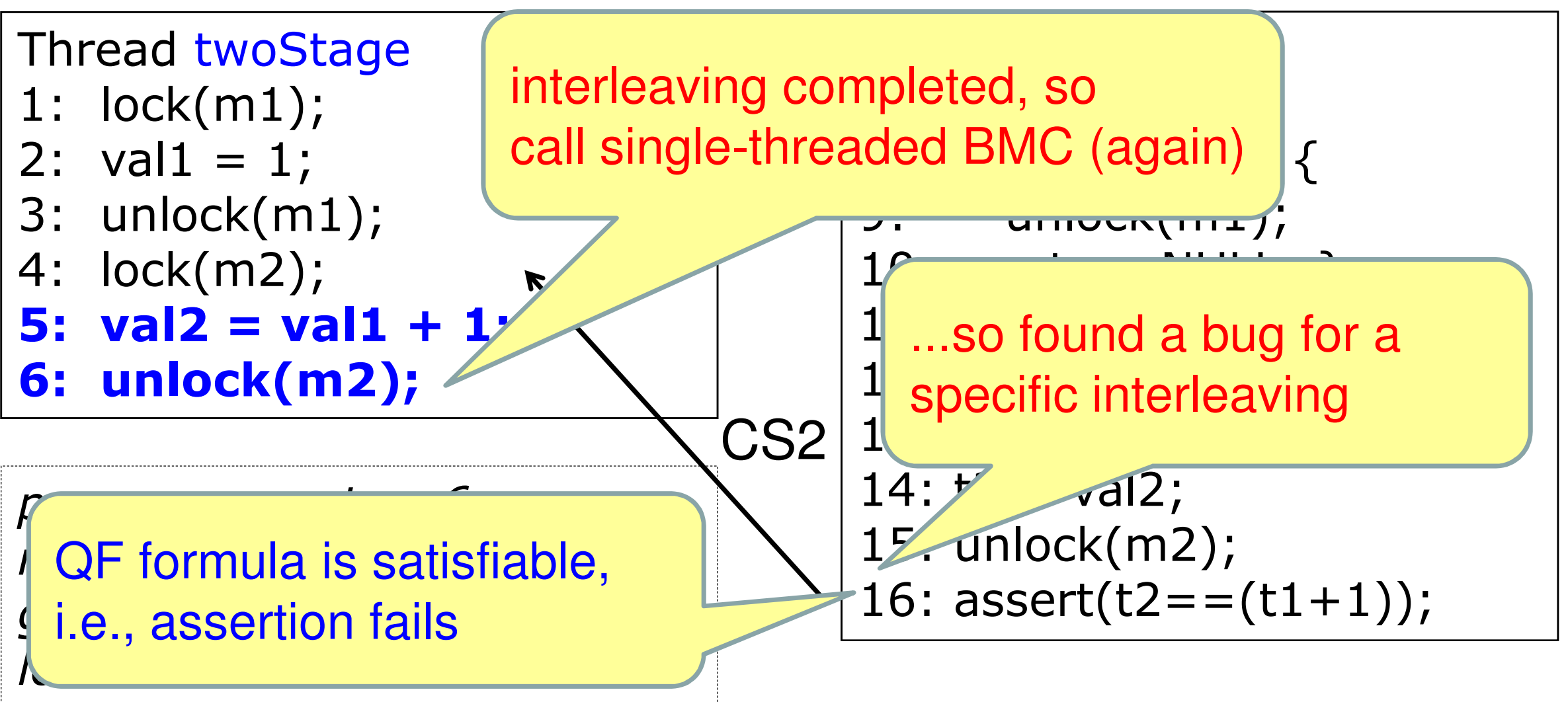




\section{Lazy exploration of interleavings}

Idea: iteratively generate all possible interleavings and call the BMC procedure on each interleaving

... combines

- symbolic model checking: on each individual interleaving

- explicit state model checking: explore all interleavings 


\section{Lazy exploration of interleavings - Reachability Tree}

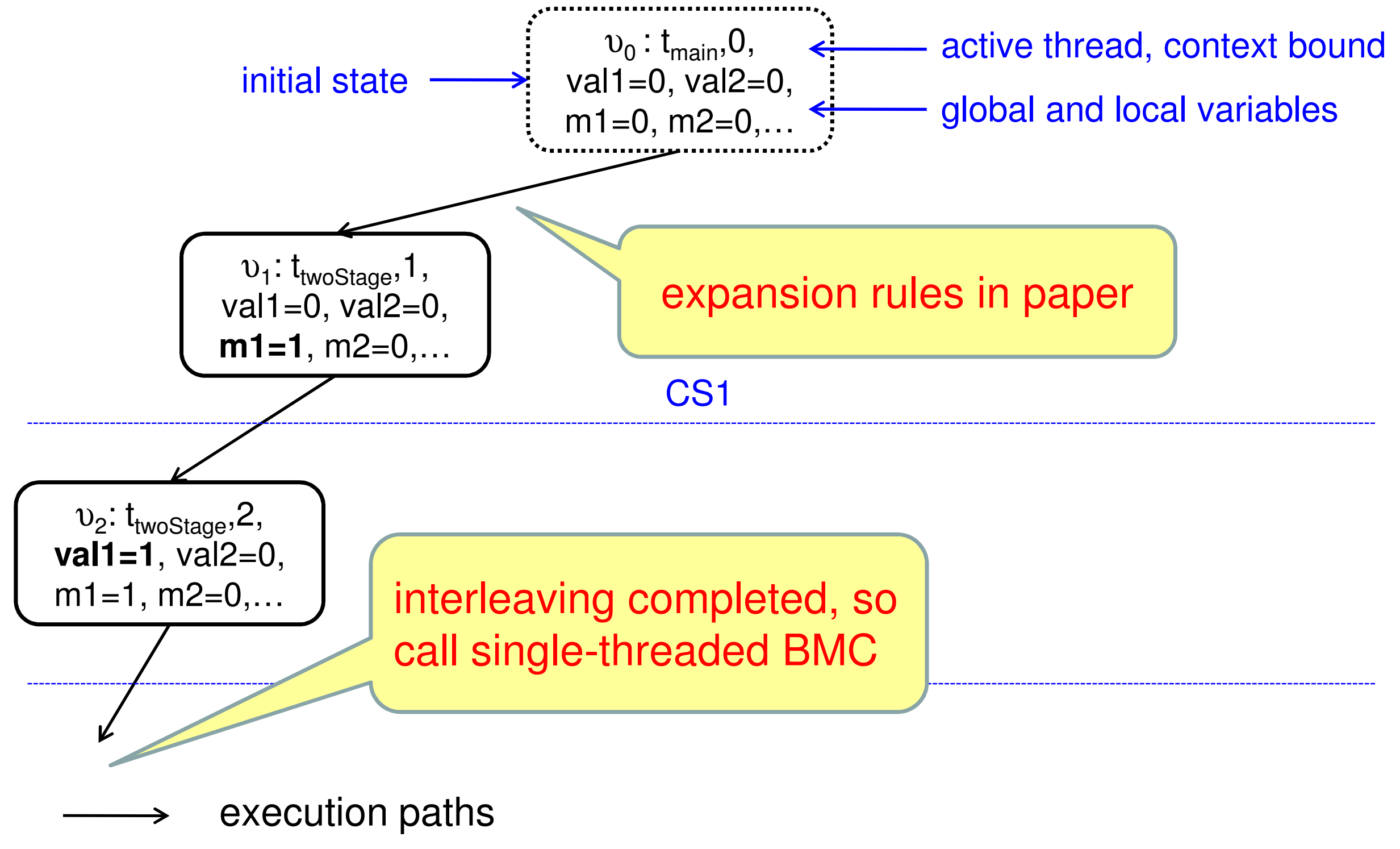




\section{Lazy exploration of interleavings - Reachability Tree}

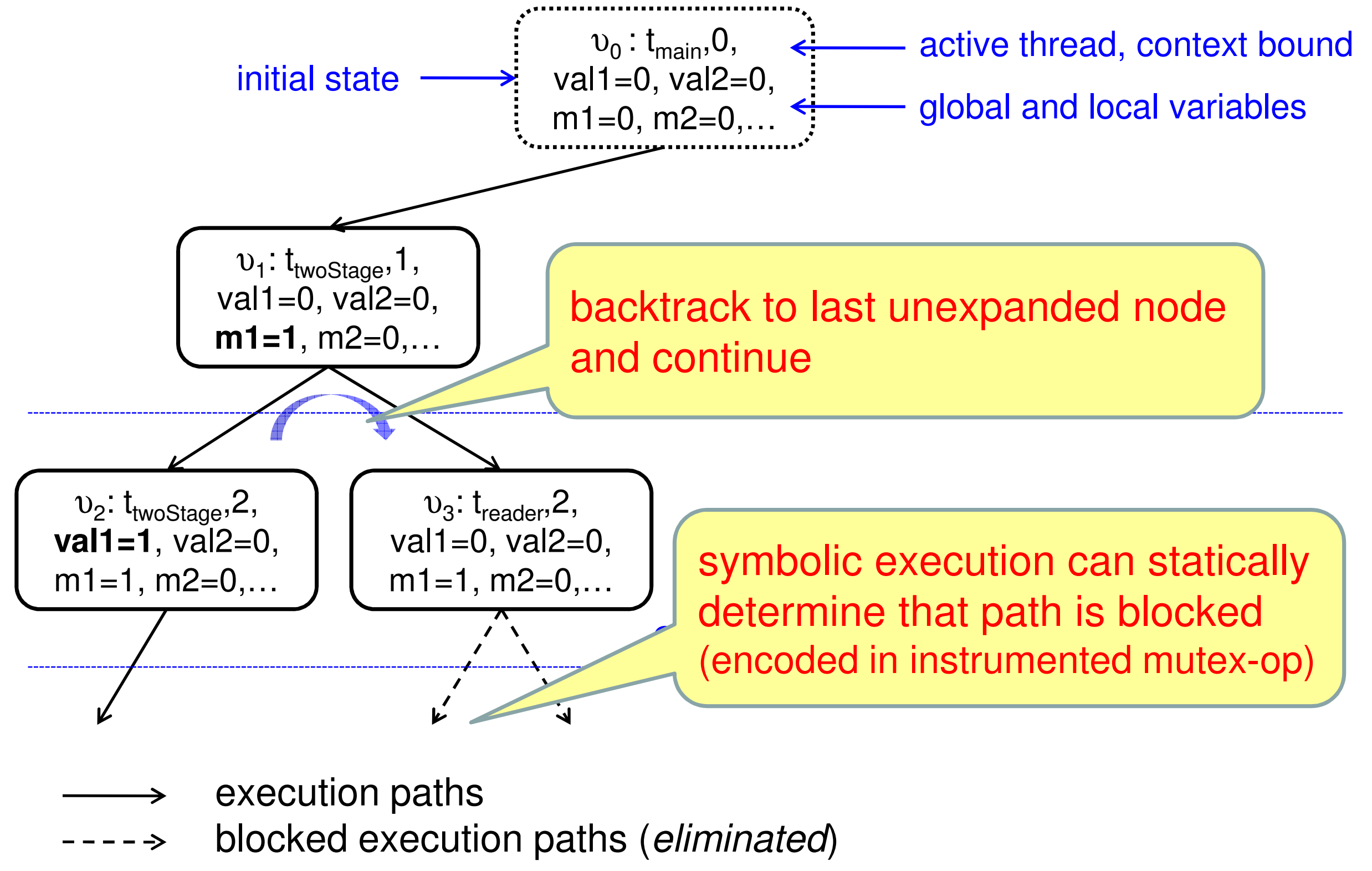




\section{Lazy exploration of interleavings - Reachability Tree}

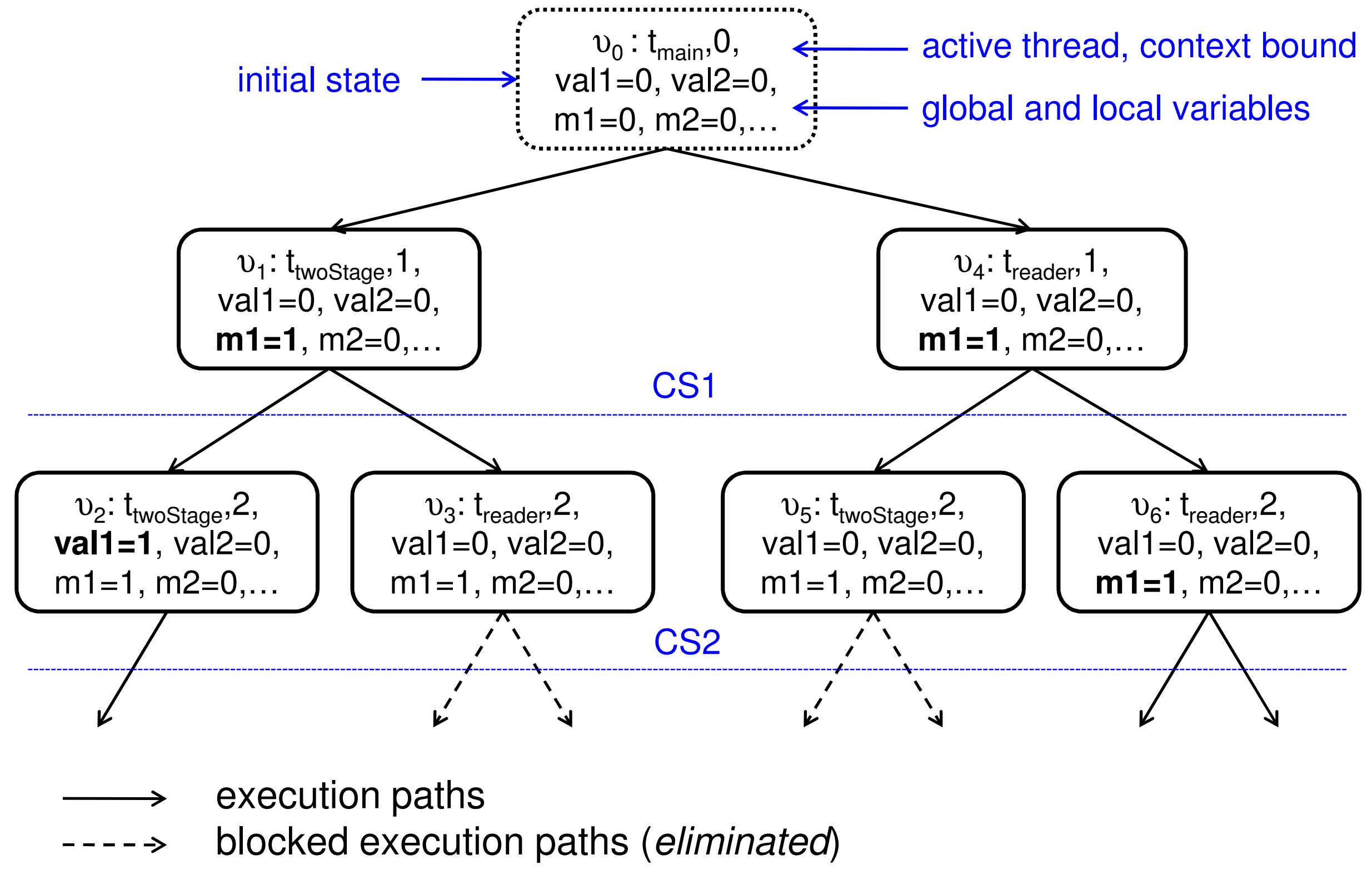




\section{Lazy approach is naïve but useful}

- bugs usually manifest in few context switches [Qadeer\&Rehof'05]

- bound the number of context switches allowed per thread

- number of executions: $O\left(n^{c}\right)$

- exploit which transitions are enabled in a given state

- reduces number of executions

- keep in memory the parent nodes of all unexplored paths only

- each formula corresponds to one possible interleaving only, its size is relatively small

- ... but can suffer performance degradation:

- in particular for correct programs where we need to invoke the SMT solver once for each possible execution path 


\section{Schedule Recording}

Idea: systematically encode all possible interleavings into one formula

- explore reachability tree in same way as lazy approach

- ... but call SMT solver only once

- add a schedule guard $t s_{i}$ for each context switch block $i$

$\left(0<\mathrm{ts}_{i} \leq\right.$ \#threads $)$

- record in which order the scheduler has executed the program

- SMT solver determines the order in which threads are simulated

- add scheduler guards only to effective statements (assignments and assertions)

- record effective context switches (ECS)

- ECS block: sequence of program statements that are executed with no intervening ECS 


\section{Schedule Recording - Interleaving \#1}

statements:

twoStage-ECS:

reader-ECS:

Thread twoStage

1 : lock $(\mathrm{m} 1)$;

2: vali $=1$;

3: unlock (m1);

4: $\operatorname{lock}(\mathrm{m} 2)$;

5: val2 = val1 $+1 ;$

6: unlock $(\mathrm{m} 2)$;

\section{ECS block}

8: if $($ val1 $==0)\{$

9: $\operatorname{unlock}(\mathrm{m} 1)$;

10: return NULL; $\}$

11: $\mathrm{t} 1$ = val1;

12: $\operatorname{unlock}(\mathrm{m} 1)$;

13: $\operatorname{lock}(\mathrm{m} 2)$;

14: $\mathrm{t2}=\mathrm{val2}$;

15: $\operatorname{unlock}(\mathrm{m} 2)$;

16: $\operatorname{assert}(\mathrm{t} 2==(\mathrm{t} 1+1))$; 


\section{Schedule Recording - Interleaving \#1}

statements: 1

twoStage-ECS: $(1,1)$

guarded statement can only be

executed if statement 1 is

reader-ECS: scheduled in ECS block 1

Thread twoStage

1: $\operatorname{lock}(\mathrm{m} 1)$;

2: val1 $=1$;

3: unlock $(\mathrm{m} 1)$;

4: $\operatorname{lock}(\mathrm{m} 2)$;

5: val2 $=$ val1 +1 ;

6: unlock $(\mathrm{m} 2)$;

Thread reader

7. 1.-1.n+1.

each program statement is then prefixed by a schedule guard $t s_{i}=j$, where:

- $i$ is the ECS block number

- $j$ is the thread identifier 1... . .....,

15: unlock $(\mathrm{m} 2)$;

16: $\operatorname{assert}(\mathrm{t} 2==(\mathrm{t} 1+1))$; 


\section{Schedule Recording - Interleaving \#1}

statements: 1-2

twoStage-ECS: $(1,1)-(2,2)$

reader-ECS:

Thread twoStage
1: $\operatorname{lock}(\mathrm{m} 1) ; \quad t s_{1}==1$
2: $\operatorname{val1}=1 ; \quad t s_{2}==1$
3: $\operatorname{unlock}(\mathrm{m} 1) ;$
4: $\operatorname{lock}(\mathrm{m} 2) ;$
5: $\operatorname{val} 2=\operatorname{val} 1+1 ;$
6: $\operatorname{unlock}(\mathrm{m} 2) ;$
Thread reader
7: $\operatorname{lock}(\mathrm{m} 1)$;
8: if (val1 $==0)\{$
9: $\operatorname{unlock}(\mathrm{m} 1)$;
10: return NULL; \}
11: $\mathrm{t} 1$ = val1;
12: unlock $(\mathrm{m} 1)$;
13: $\operatorname{lock}(\mathrm{m} 2)$;
14: $\mathrm{t} 2$ = val2;
15: unlock $(\mathrm{m} 2)$;
16: $\operatorname{assert}(\mathrm{t} 2==(\mathrm{t} 1+1))$; 


\section{Schedule Recording - Interleaving \#1}

statements: 1-2-3

twoStage-ECS: $(1,1)-(2,2)-(3,3)$

reader-ECS:

Thread twoStage

1: $\operatorname{lock}(\mathrm{m} 1) ; \quad t \mathrm{~s}_{1}==1$

2: val1 $=1 ; \quad t s_{2}==1$

3: $\operatorname{unlock}(\mathrm{m} 1) ; \quad t s_{3}==1$

4: $\operatorname{lock}(\mathrm{m} 2)$;

5: val2 = val1 +1 ;

6: unlock (m2);
Thread reader

7: $\operatorname{lock}(\mathrm{m} 1)$;

8: if (val1 $==0)\{$

9: $\operatorname{unlock}(\mathrm{m} 1)$;

10: return NULL; $\}$

11: $\mathrm{t} 1$ = val1;

12: $\operatorname{unlock}(\mathrm{m} 1)$;

13: $\operatorname{lock}(\mathrm{m} 2)$;

14: $\mathrm{t} 2$ = val2;

15: unlock $(\mathrm{m} 2)$;

16: $\operatorname{assert}(\mathrm{t} 2==(\mathrm{t} 1+1))$; 


\section{Schedule Recording - Interleaving \#1}

statements: 1-2-3-7

twoStage-ECS: $(1,1)-(2,2)-(3,3)$

reader-ECS: $(7,4)$

Thread twoStage

1: $\operatorname{lock}(\mathrm{m} 1)$;

2: val1 = 1 ;

3: $\operatorname{unlock}(\mathrm{m} 1)$;

4: $\operatorname{lock}(\mathrm{m} 2)$;

5: val2 = val1 +1 ;

6: unlock (m2);

$$
\begin{aligned}
& t s_{1}==1 \text { CS } \begin{array}{l}
\text { Thread reader } \\
7: \operatorname{lock}(\mathrm{m} 1)
\end{array} \\
& t s_{2}==1 \quad 8: \text { if }(\text { val1 }==0)\{ \\
& \left.t s_{3}=1\right\} \quad \text { 9: } \operatorname{unlock}(\mathrm{m} 1) \text {; } \\
& \text { 10: return NULL; \} } \\
& \text { 11: } \mathrm{t} 1 \text { = val1; } \\
& \text { 12: unlock(m1); } \\
& \text { 13: } \operatorname{lock}(\mathrm{m} 2) \text {; } \\
& \text { 14: t2 = val2; } \\
& \text { 15: unlock }(\mathrm{m} 2) \text {; } \\
& \text { 16: } \operatorname{assert}(\mathrm{t} 2==(\mathrm{t} 1+1)) \text {; }
\end{aligned}
$$




\section{Schedule Recording - Interleaving \#1}

statements: 1-2-3-7-8

twoStage-ECS: $(1,1)-(2,2)-(3,3)$

reader-ECS: $(7,4)-(8,5)$

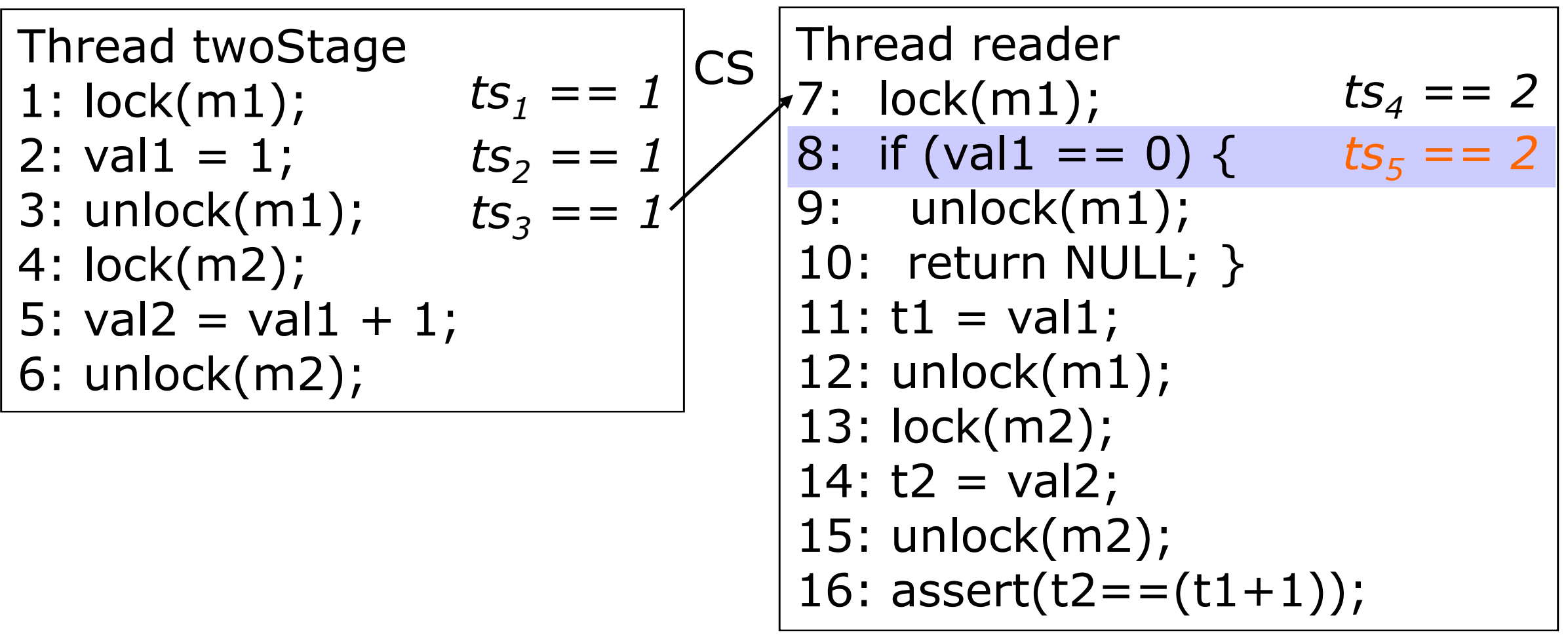




\section{Schedule Recording - Interleaving \#1}

statements: 1-2-3-7-8-11

twoStage-ECS: $(1,1)-(2,2)-(3,3)$

reader-ECS: $(7,4)-(8,5)-(11,6)$

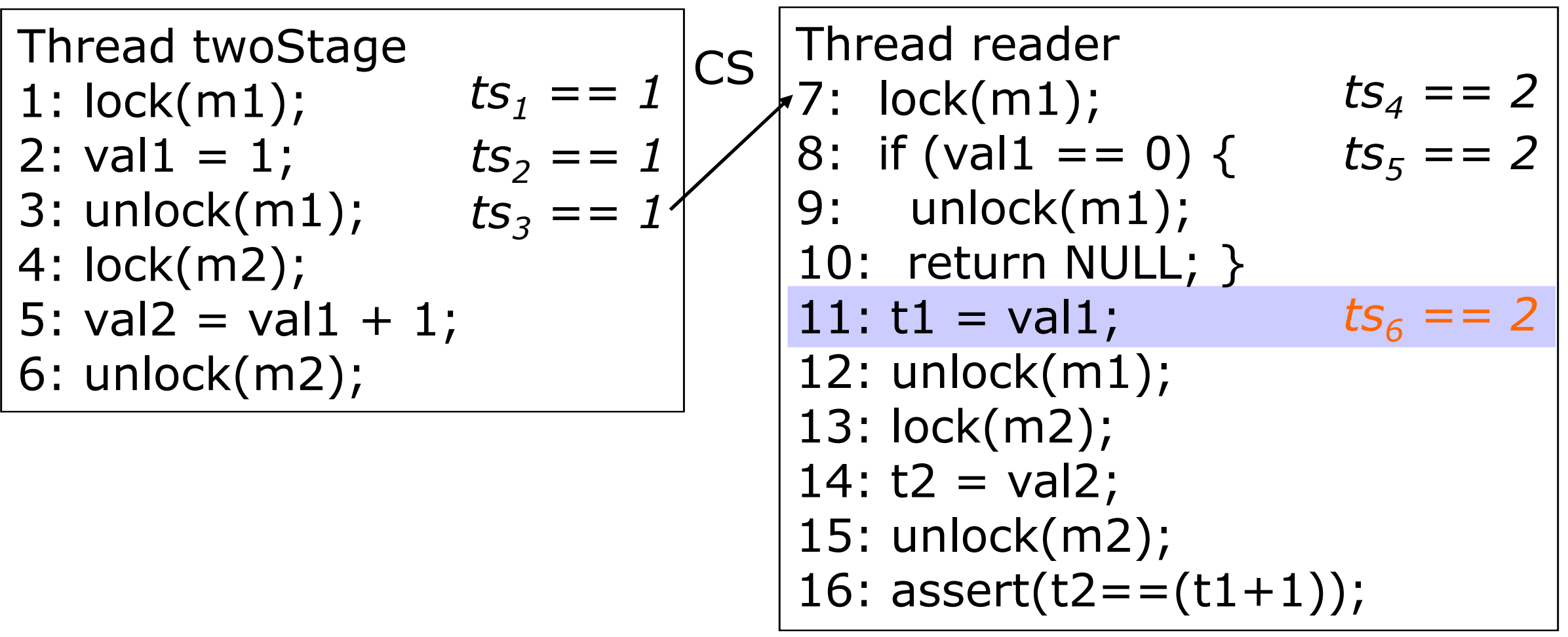




\section{Schedule Recording - Interleaving \#1}

statements: 1-2-3-7-8-11-12

twoStage-ECS: $(1,1)-(2,2)-(3,3)$

reader-ECS: $(7,4)-(8,5)-(11,6)-(12,7)$

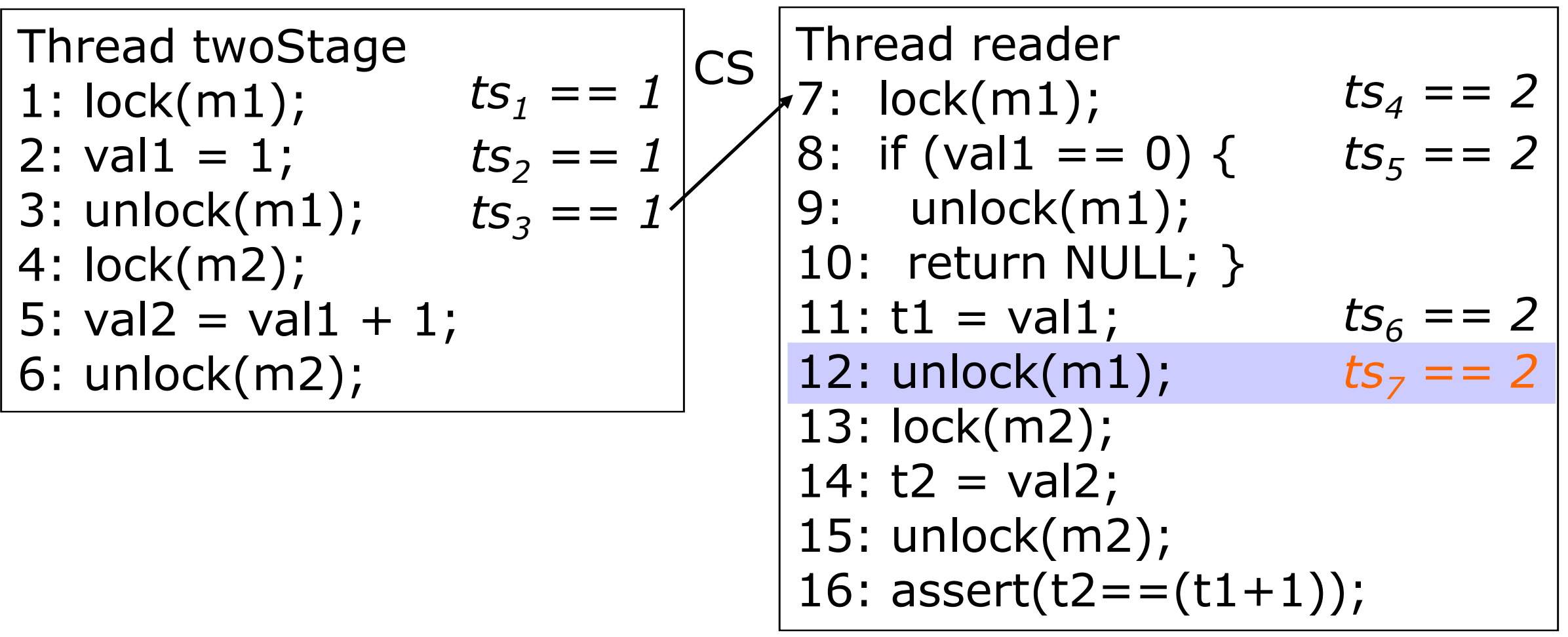




\section{Schedule Recording - Interleaving \#1}

statements: 1-2-3-7-8-11-12-4

twoStage-ECS: $(1,1)-(2,2)-(3,3)-(4,8)$

reader-ECS: $(7,4)-(8,5)-(11,6)-(12,7)$

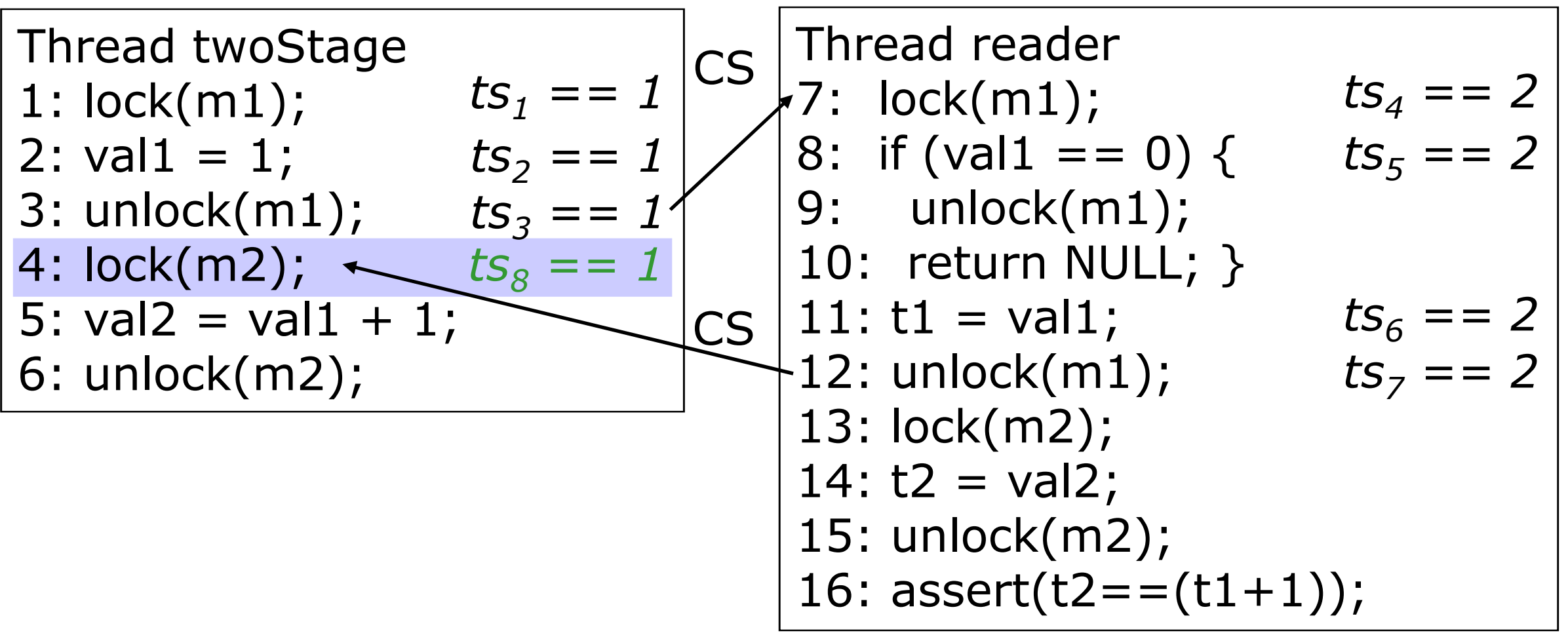




\section{Schedule Recording - Interleaving \#1}

statements: 1-2-3-7-8-11-12-4-5

twoStage-ECS: $(1,1)-(2,2)-(3,3)-(4,8)-(5,9)$

reader-ECS: $(7,4)-(8,5)-(11,6)-(12,7)$

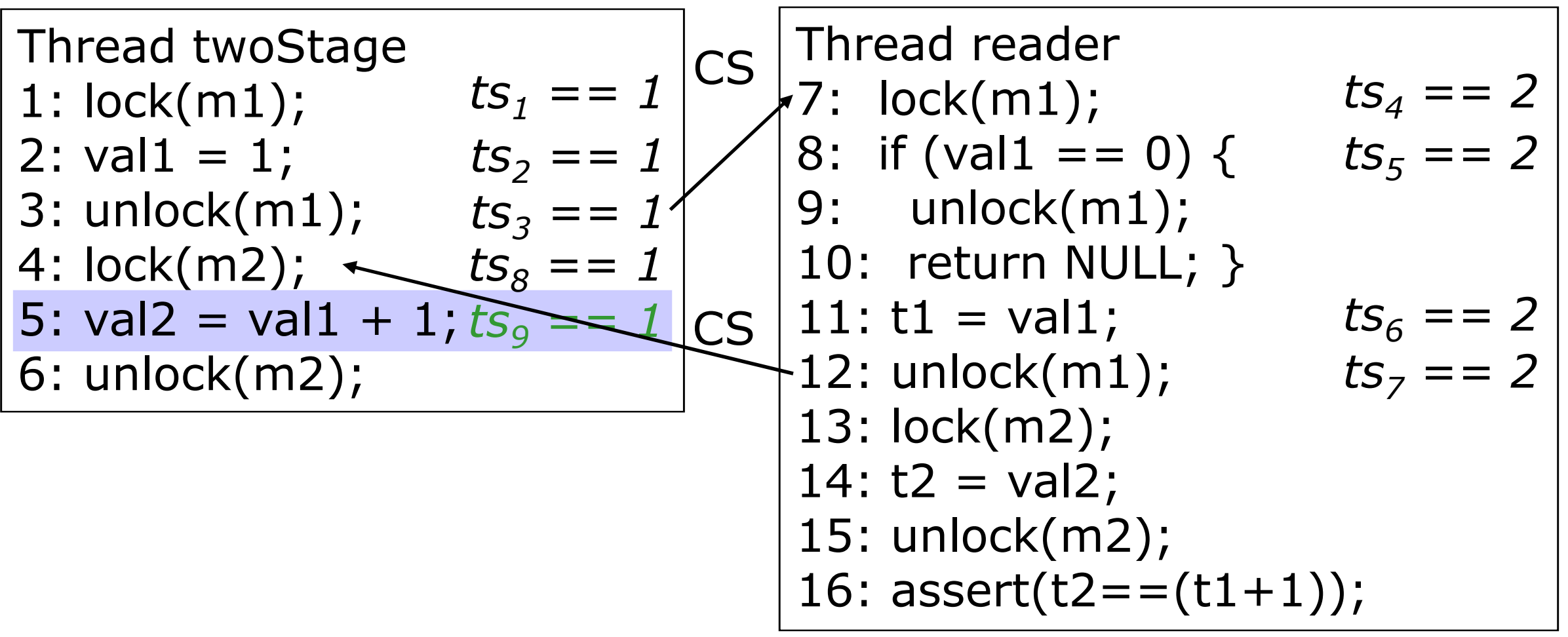




\section{Schedule Recording - Interleaving \#1}

statements: 1-2-3-7-8-11-12-4-5-6

twoStage-ECS: $(1,1)-(2,2)-(3,3)-(4,8)-(5,9)-(6,10)$

reader-ECS: $(7,4)-(8,5)-(11,6)-(12,7)$

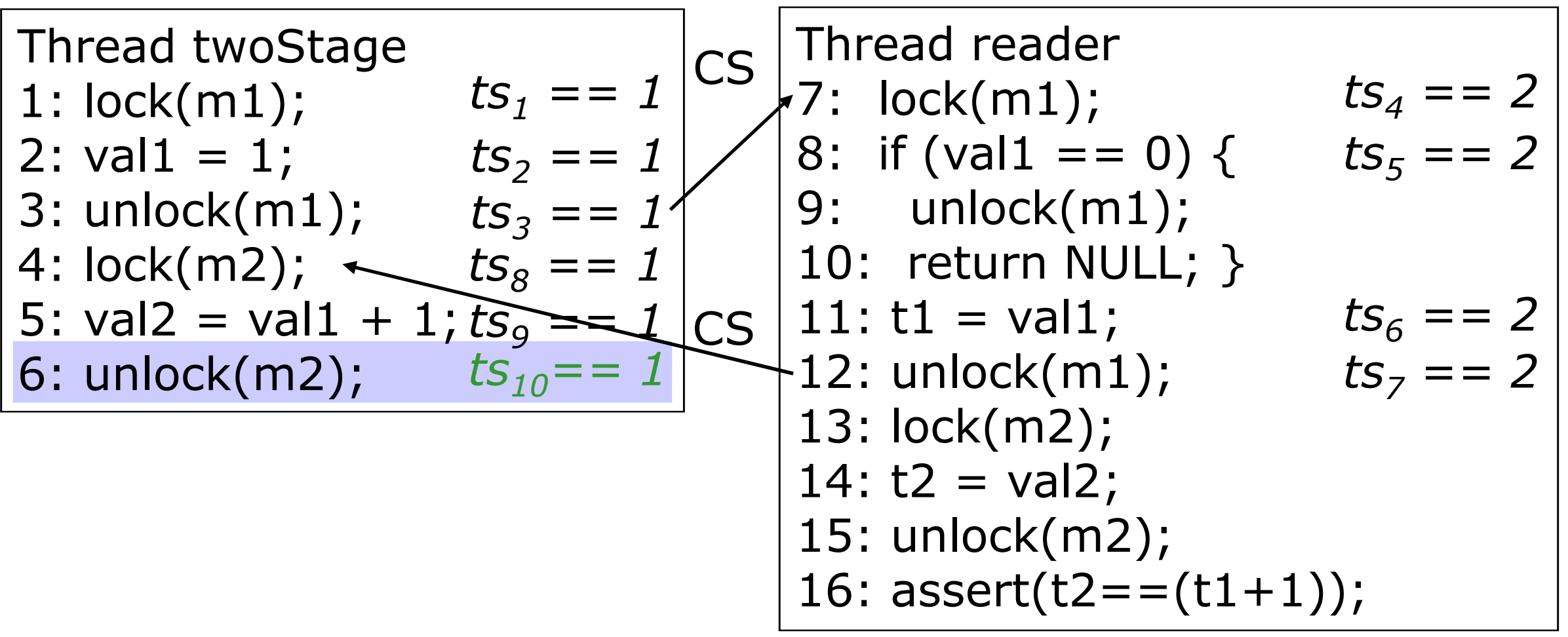




\section{Schedule Recording - Interleaving \#1}

statements: 1-2-3-7-8-11-12-4-5-6-13

twoStage-ECS: $(1,1)-(2,2)-(3,3)-(4,8)-(5,9)-(6,10)$

reader-ECS: $(7,4)-(8,5)-(11,6)-(12,7)-(13,11)$

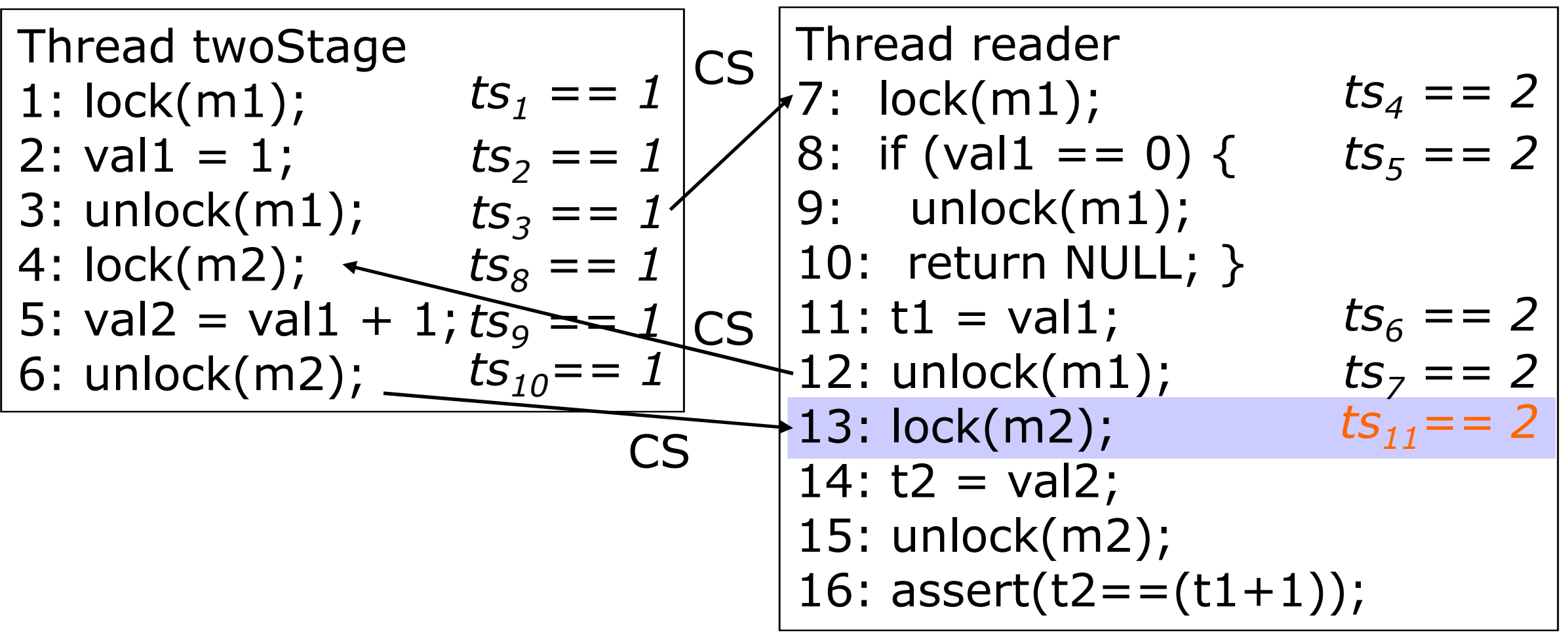




\section{Schedule Recording - Interleaving \#1}

statements: 1-2-3-7-8-11-12-4-5-6-13-14

twoStage-ECS: $(1,1)-(2,2)-(3,3)-(4,8)-(5,9)-(6,10)$

reader-ECS: $(7,4)-(8,5)-(11,6)-(12,7)-(13,11)-(14,12)$

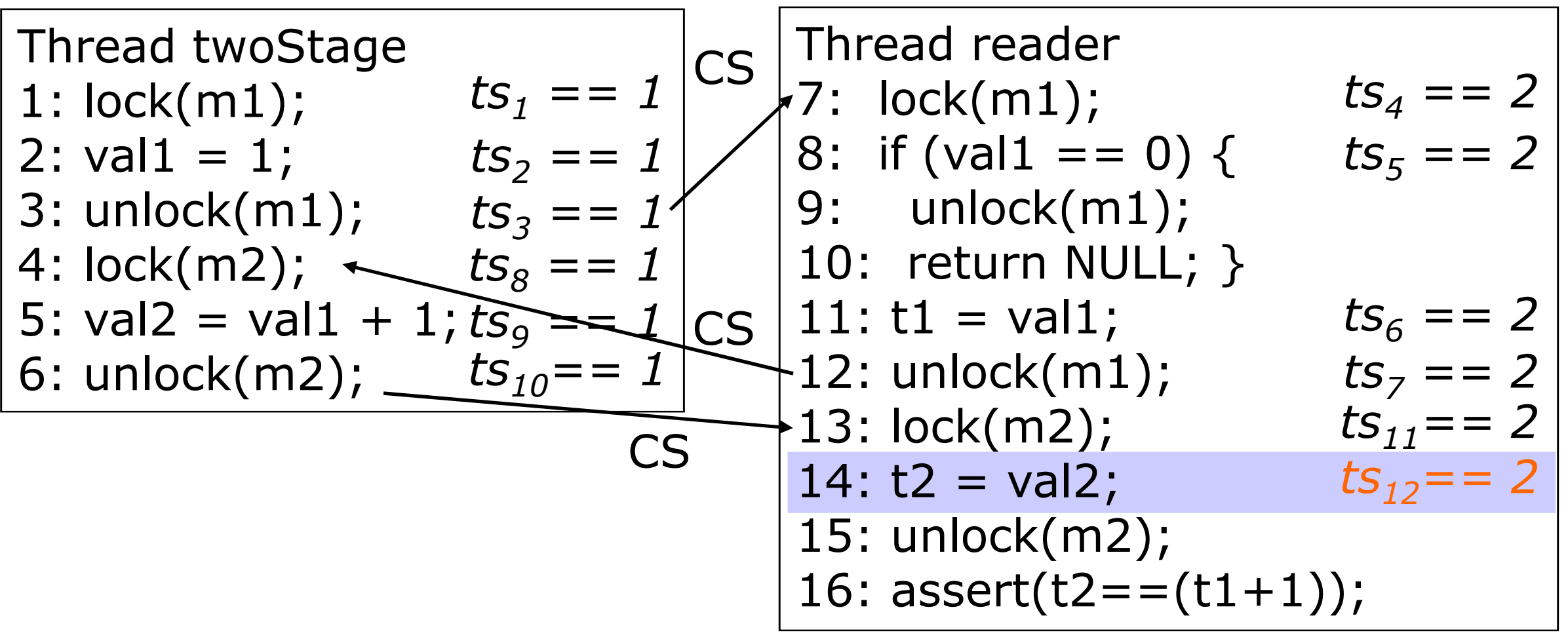




\section{Schedule Recording - Interleaving \#1}

statements: 1-2-3-7-8-11-12-4-5-6-13-14-15

twoStage-ECS: $(1,1)-(2,2)-(3,3)-(4,8)-(5,9)-(6,10)$

reader-ECS: $(7,4)-(8,5)-(11,6)-(12,7)-(13,11)-(14,12)-(15,13)$

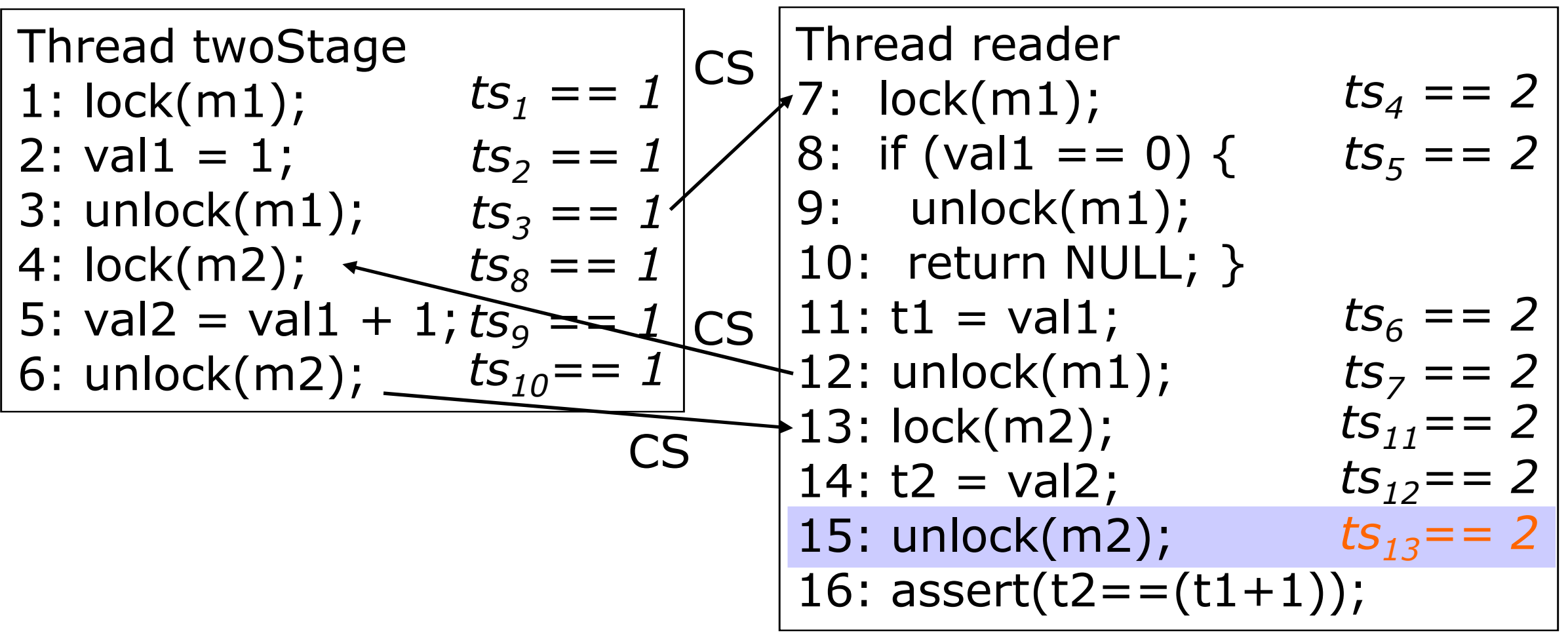




\section{Schedule Recording - Interleaving \#1}

statements: 1-2-3-7-8-11-12-4-5-6-13-14-15-16

twoStage-ECS: $(1,1)-(2,2)-(3,3)-(4,8)-(5,9)-(6,10)$

reader-ECS: $(7,4)-(8,5)-(11,6)-(12,7)-(13,11)-(14,12)-(15,13)-(16,14)$

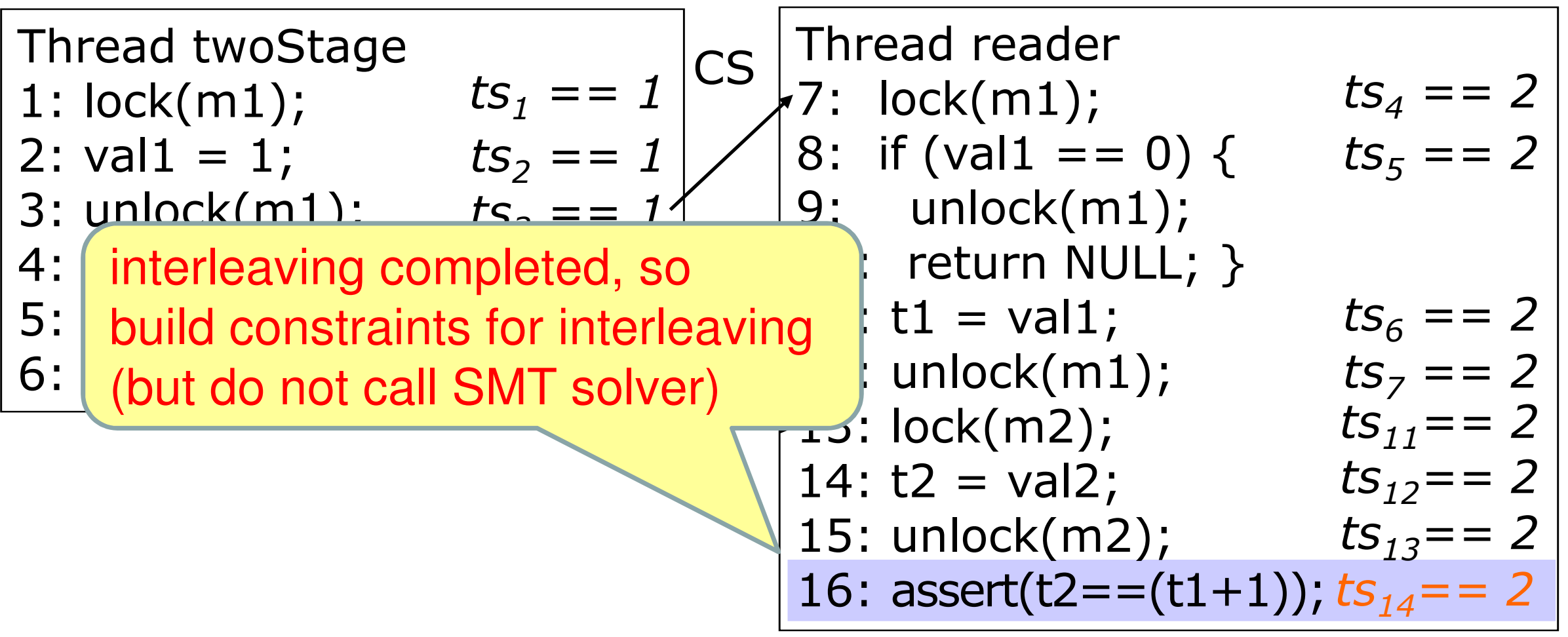




\section{Schedule Recording - Interleaving \#2}

statements: 1-2-3-7-8-11-12-13-14-15-16-4-5-6

twoStage-ECS: $(1,1)-(2,3)-(3,4)-(4,12)-(5,13)-(6,14)$

reader-ECS: $(7,4)-(8,5)-(11,6)-(12,7)-(13,8)-(14,9)-(15,10)-(16,11)$

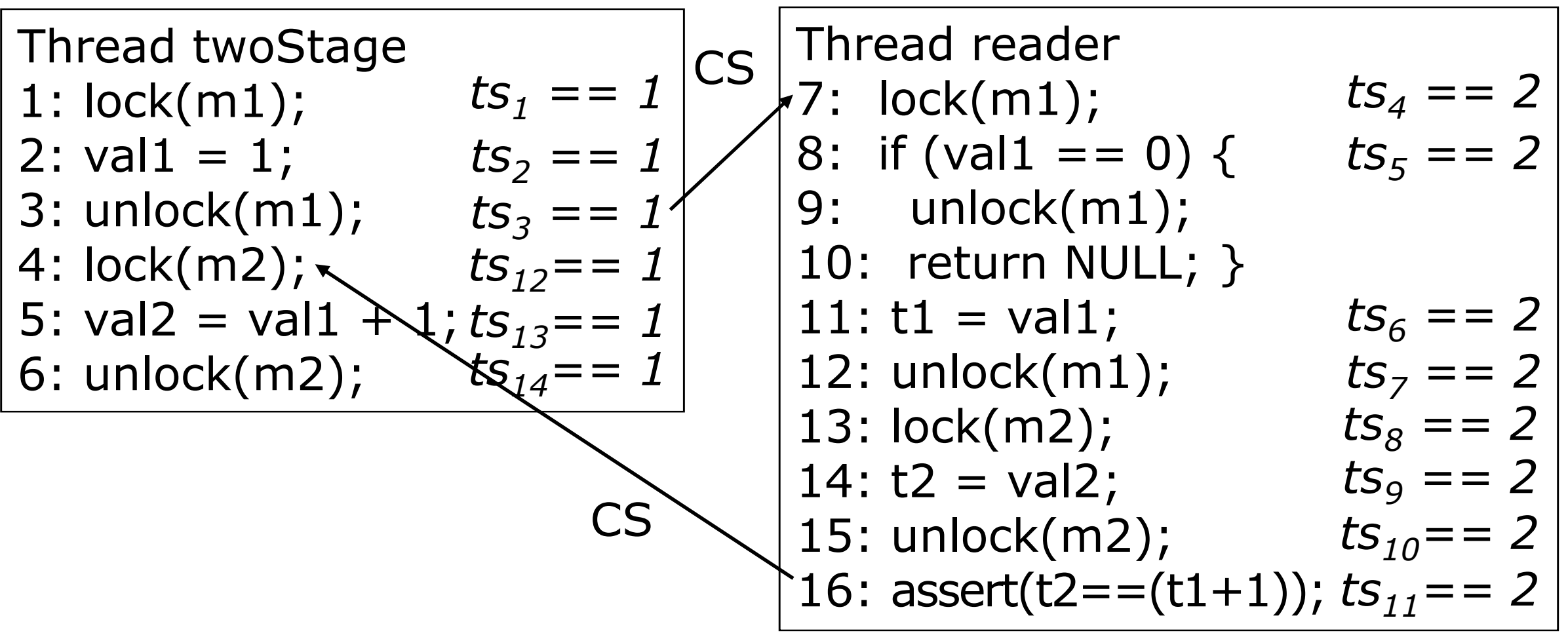




\section{Schedule Recording: Execution Paths}

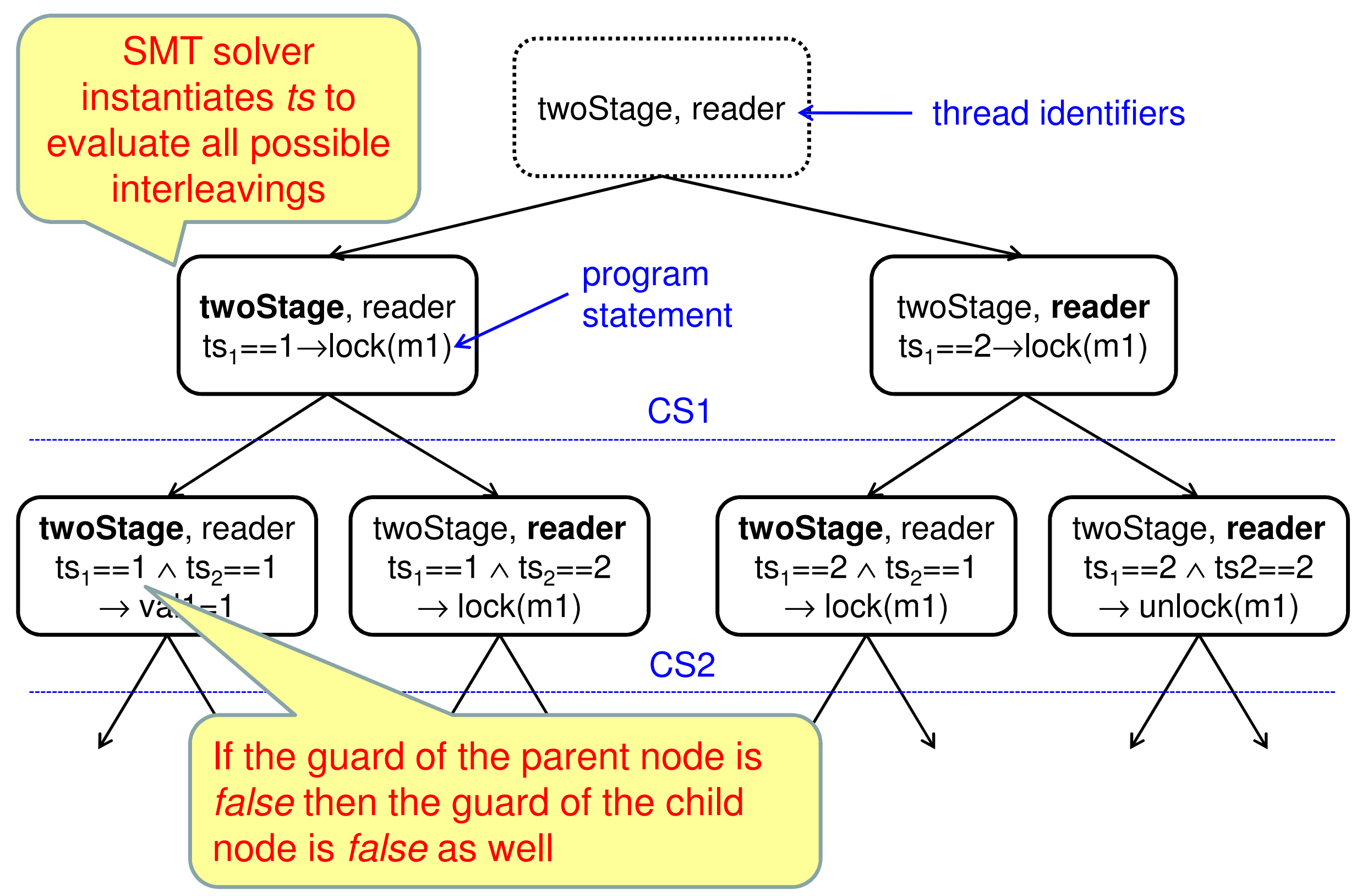




\section{Observations about the schedule recoding approach}

- systematically explore the thread interleavings as before, but:

- add schedule guards to record in which order the scheduler has executed the program

- encode all execution paths into one formula

$\triangleright$ bound the number of context switches

$\triangleright$ exploit which transitions are enabled in a given state

- number of threads and context switches grows very large quickly, and easily "blow-up" the solver:

- there is a clear trade-off between usage of time and memory resources 


\section{Under-approximation and Widening}

Idea: check models with an increased set of allowed interleavings [Grumberg\&et al.'05]

- start from a single interleaving (under-approximation) and widen the model by adding more interleavings incrementally

Main steps of the algorithm:

1. encode control literals $\left(\mathrm{cl}_{\mathrm{i}, \mathrm{j}}\right)$ into the verification condition $\psi$ $\triangleright \mathrm{Cl}_{\mathrm{i}, \mathrm{j}}$ where $i$ is the ECS block number and $j$ is the thread identifier

2. check the satisfiability of $\psi$ (stop if $\psi$ is satisfiable)

3. extract proof objects generated by the SMT solver

4. check whether the proof depends on the control literals (stop if the proof does not depend on the control literals)

5. remove literals that participated in the proof and go to step 2 


\section{UW Approach: Running Example}

- use the same guards as in the schedule recording approach as control literals

- but here the schedule is updated based on the information extracted from the proof

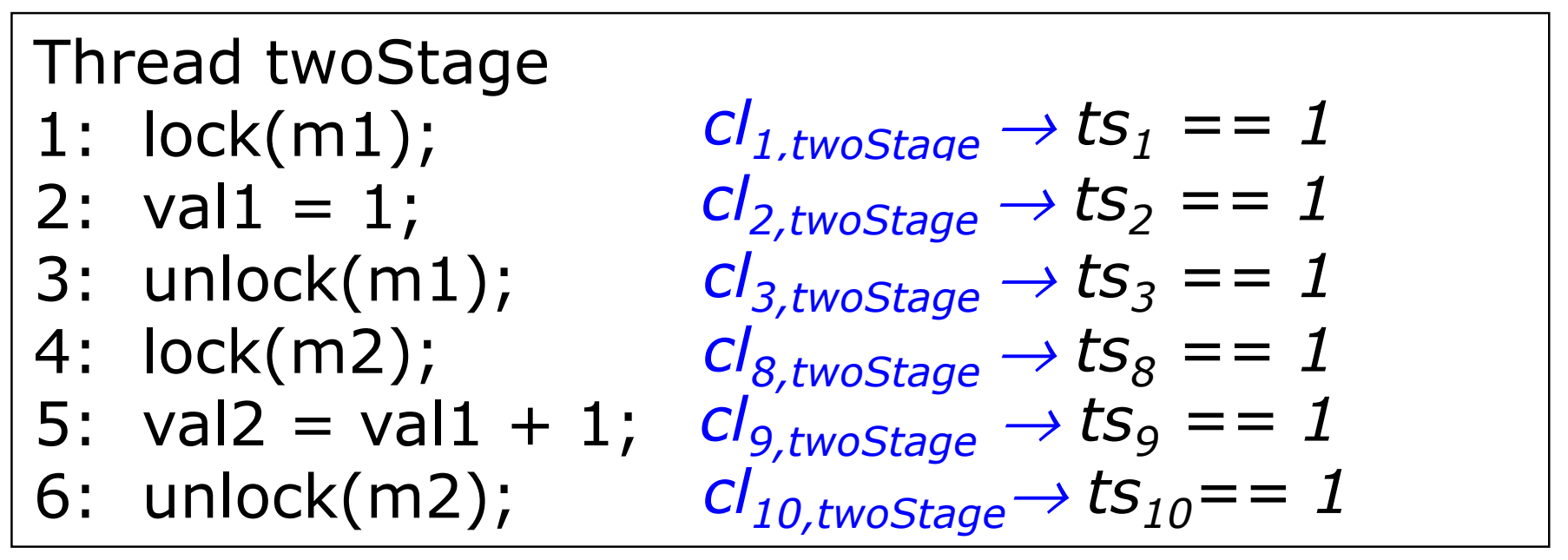

- reduce the number of control points from $m \times n$ to $e \times n$

- $m$ is the number of program statements; $n$ is the number of threads, and $e$ is the number of ECS blocks 


\section{Evaluation}




\section{Comparison of the Approaches}

- Goal: compare efficiency of the proposed approaches

- lazy exploration

- schedule recording

- underapproximation and widening

- Set-up:

- ESBMC v1.15.1 together with the SMT solver Z3 v2.11

- support the logics QF_AUFBV and QF_AUFLIRA

- standard desktop PC, time-out 3600 seconds 


\section{About the benchmarks}

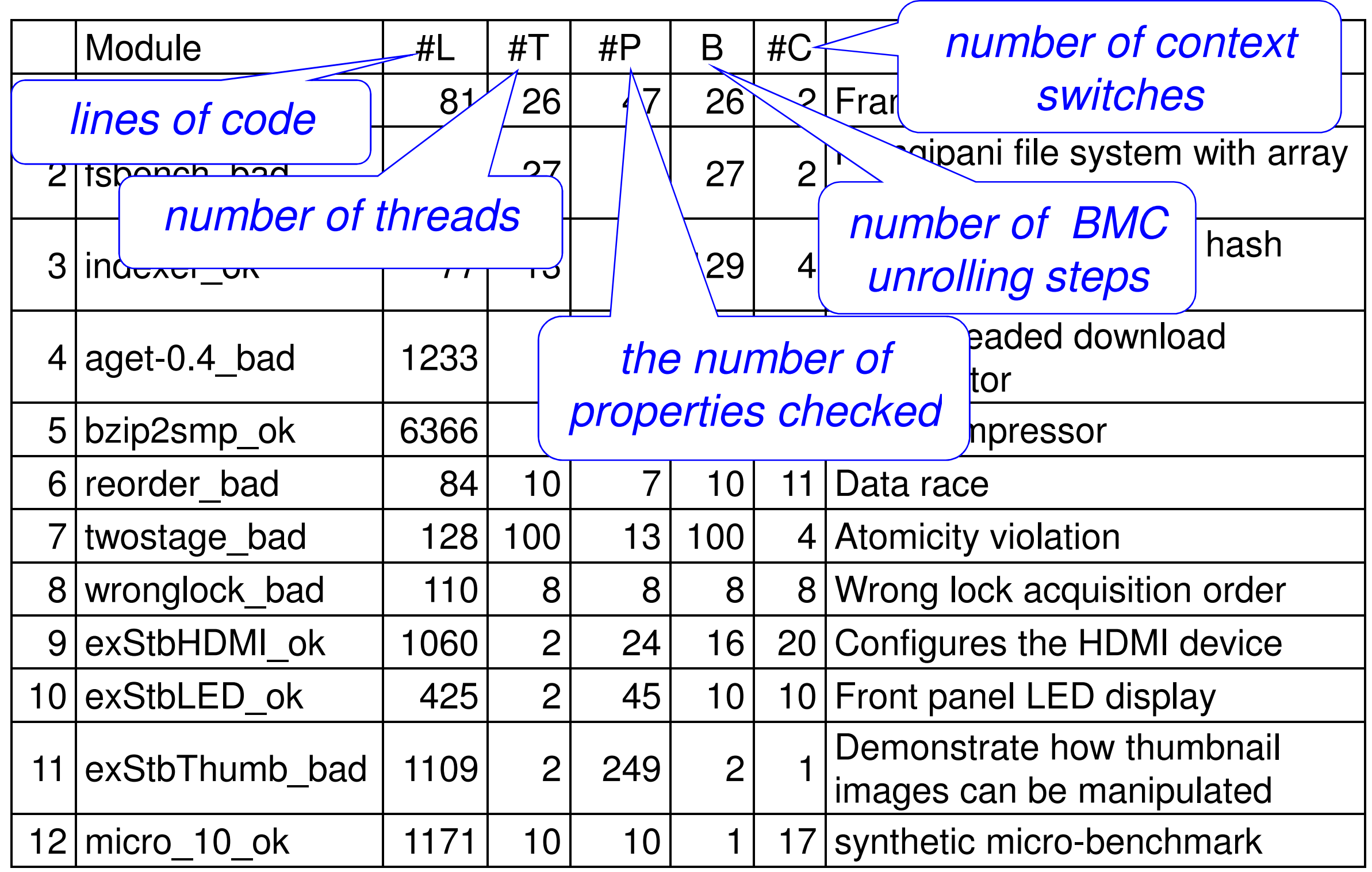




\section{About the benchmarks}

\begin{tabular}{|c|c|c|c|c|c|c|c|}
\hline & Module & \multirow{2}{*}{ \# } & \multirow{3}{*}{\multicolumn{3}{|c|}{$\begin{array}{c}\text { Inspect } \\
\text { benchmark } \\
\text { suite }\end{array}$}} & C & Description \\
\hline 1 & fsbench_ok & & & & & 2 & Frangipani file system \\
\hline 2 & fsbench_bad & & & & & 2 & $\begin{array}{l}\text { Frangipani file system with } \\
\text { array out of bounds }\end{array}$ \\
\hline 3 & indexer_ok & 77 & 13 & 21 & 129 & 4 & $\begin{array}{l}\text { Insert messages into a hash } \\
\text { table concurrently }\end{array}$ \\
\hline 4 & aget-0.4_bad & 1233 & 3 & 279 & 200 & 2 & $\begin{array}{l}\text { Multi-threaded download } \\
\text { accelerator }\end{array}$ \\
\hline 5 & bzip2smp_ok & 6366 & 3 & 8568 & 1 & 9 & Data compressor \\
\hline 6 & reorder_bad & 84 & 10 & 7 & 10 & 11 & Data race \\
\hline 7 & twostage_bad & 128 & 100 & 13 & 100 & 4 & Atomicity violation \\
\hline 8 & wronglock_bad & 110 & 8 & 8 & 8 & 8 & Wrong lock acquisition order \\
\hline 9 & exStbHDMI_ok & 1060 & 2 & 24 & 16 & 20 & Configures the HDMI device \\
\hline 10 & exStbLED_ok & 425 & 2 & 45 & 10 & 10 & Front panel LED display \\
\hline 11 & exStbThumb_bad & 1109 & 2 & 249 & 2 & 1 & $\begin{array}{l}\text { Demonstrate how thumbnail } \\
\text { images can be manipulated }\end{array}$ \\
\hline 12 & micro_10_ok & 1171 & 10 & 10 & 1 & 17 & synthetic micro-benchmark \\
\hline
\end{tabular}




\section{About the benchmarks}

\begin{tabular}{|c|c|c|c|c|c|c|c|}
\hline & Module & \#L & $\overline{\# T}$ & \#P & $B$ & \#C & Description \\
\hline 1 & fsbench_ok & 81 & 26 & 47 & 26 & 2 & Frangipani file system \\
\hline 2 & fsbench_bad & 80 & 27 & 48 & 27 & 2 & $\begin{array}{l}\text { Frangipani file system with array } \\
\text { out of bounds }\end{array}$ \\
\hline 3 & indexer_ok & \multirow{2}{*}{\multicolumn{4}{|c|}{$\begin{array}{c}\text { VV-lab } \\
\text { benchmark } \\
\text { suite }\end{array}$}} & 4 & $\begin{array}{l}\text { Insert messages into a hash } \\
\text { table concurrently }\end{array}$ \\
\hline 4 & aget-0.4_bad & & & & & 2 & $\begin{array}{l}\text { Multi-threaded download } \\
\text { accelerator }\end{array}$ \\
\hline 5 & bzip2smp_ok & & 3 & 8568 & 1 & 9 & Data compressor \\
\hline 6 & reorder_bad & 84 & 10 & 7 & 10 & 11 & Data race \\
\hline 7 & twostage_bad & 128 & 100 & 13 & 100 & 4 & Atomicity violation \\
\hline 8 & wronglock_bad & 110 & 8 & 8 & 8 & 8 & Wrong lock acquisition order \\
\hline 9 & exStbHDMI_ok & 1060 & 2 & 24 & 16 & 20 & Configures the HDMI device \\
\hline 10 & exStbLED_ok & 425 & 2 & 45 & 10 & 10 & Front panel LED display \\
\hline 11 & exStbThumb_bad & 1109 & 2 & 249 & 2 & 1 & $\begin{array}{l}\text { Demonstrate how thumbnail } \\
\text { images can be manipulated }\end{array}$ \\
\hline 12 & micro_10_ok & 1171 & 10 & 10 & 1 & 17 & synthetic micro-benchmark \\
\hline
\end{tabular}




\section{About the benchmarks}

\begin{tabular}{|c|c|c|c|c|c|c|c|}
\hline & Module & \#L & \#T & \#P & $B$ & \#C & Description \\
\hline 1 & fsbench_ok & 81 & 26 & 47 & 26 & 2 & Frangipani file system \\
\hline 2 & fsbench_bad & 80 & 27 & 48 & 27 & 2 & $\begin{array}{l}\text { Frangipani file system with array } \\
\text { out of bounds }\end{array}$ \\
\hline 3 & indexer_ok & 77 & 13 & 21 & 129 & 4 & $\begin{array}{l}\text { Insert messages into a hash } \\
\text { table concurrently }\end{array}$ \\
\hline 4 & aget-0.4_bad & 1233 & 3 & 279 & 200 & 2 & $\begin{array}{l}\text { Multi-threaded download } \\
\text { accelerator }\end{array}$ \\
\hline 5 & bzip2smp_ok & \multirow{3}{*}{\multicolumn{5}{|c|}{$\begin{array}{c}\text { Set-top box } \\
\text { applications from NXP } \\
\text { semiconductors }\end{array}$}} & ata compressor \\
\hline 6 & reorder_bad & & & & & & ata race \\
\hline 7 & twostage_bad & & & & & & lomicity violation \\
\hline 8 & wronglock_bad & 1 & 6 & 8 & 8 & 8 & Wrong lock acquisition order \\
\hline 9 & exStbHDMI_ok & 0 & 2 & 24 & 16 & 20 & Configures the HDMI device \\
\hline 10 & exStbLED_ok & 425 & 2 & 45 & 10 & 10 & Front panel LED display \\
\hline 11 & exStbThumb_bad & 1109 & 2 & 249 & 2 & 1 & $\begin{array}{l}\text { Demonstrate how thumbnail } \\
\text { images can be manipulated }\end{array}$ \\
\hline 12 & micro_10_ok & 1171 & 10 & 10 & 1 & 17 & synthetic micro-benchmark \\
\hline
\end{tabular}




\section{About the benchmarks}

\begin{tabular}{|c|c|c|c|c|c|c|c|c|}
\hline & Module & \#L & \#T & \#P & $\mathrm{B}$ & $\# \mathrm{C}$ & & Description \\
\hline 1 & fsbench_ok & 81 & 26 & 47 & 26 & 2 & \multicolumn{2}{|c|}{ Frangipani file system } \\
\hline 2 & fsbench_bad & 80 & 27 & 48 & 27 & 2 & \multicolumn{2}{|c|}{$\begin{array}{l}\text { Frangipani file system with array } \\
\text { out of bounds }\end{array}$} \\
\hline 3 & indexer_ok & 77 & 13 & 21 & 129 & 4 & \multicolumn{2}{|c|}{$\begin{array}{l}\text { Insert messages into a hash } \\
\text { table concurrently }\end{array}$} \\
\hline 4 & aget-0.4_bad & 1233 & 3 & 279 & 200 & 2 & \multicolumn{2}{|c|}{$\begin{array}{l}\text { Multi-threaded download } \\
\text { accelerator }\end{array}$} \\
\hline 5 & bzip2smp_ok & \multirow{5}{*}{\multicolumn{6}{|c|}{$\begin{array}{c}\text { It is used to check the } \\
\text { scalability of multi-threaded } \\
\text { software verification tools } \\
\text { [Ghafari 2010] }\end{array}$}} & ressor \\
\hline 6 & reorder_bad & & & & & & & \\
\hline 7 & twostage_bad & & & & & & & iolation \\
\hline 8 & wronglock_bad & & & & & & & acquisition order \\
\hline 9 & exStbHDMI_ok & & & & & & & the HDMI device \\
\hline 10 & exStbLED_ok & \multicolumn{2}{|l|}{42} & & 10 & 10 & \multicolumn{2}{|c|}{ Front panel LED display } \\
\hline 11 & exStbThumb_bad & & 2 & 249 & 2 & 1 & \multicolumn{2}{|c|}{$\begin{array}{l}\text { Demonstrate how thumbnail } \\
\text { images can be manipulated }\end{array}$} \\
\hline 12 & micro_10_ok & 1171 & 10 & 10 & 1 & 17 & \multicolumn{2}{|c|}{ synthetic micro-benchmark } \\
\hline
\end{tabular}


Comparison of the appro

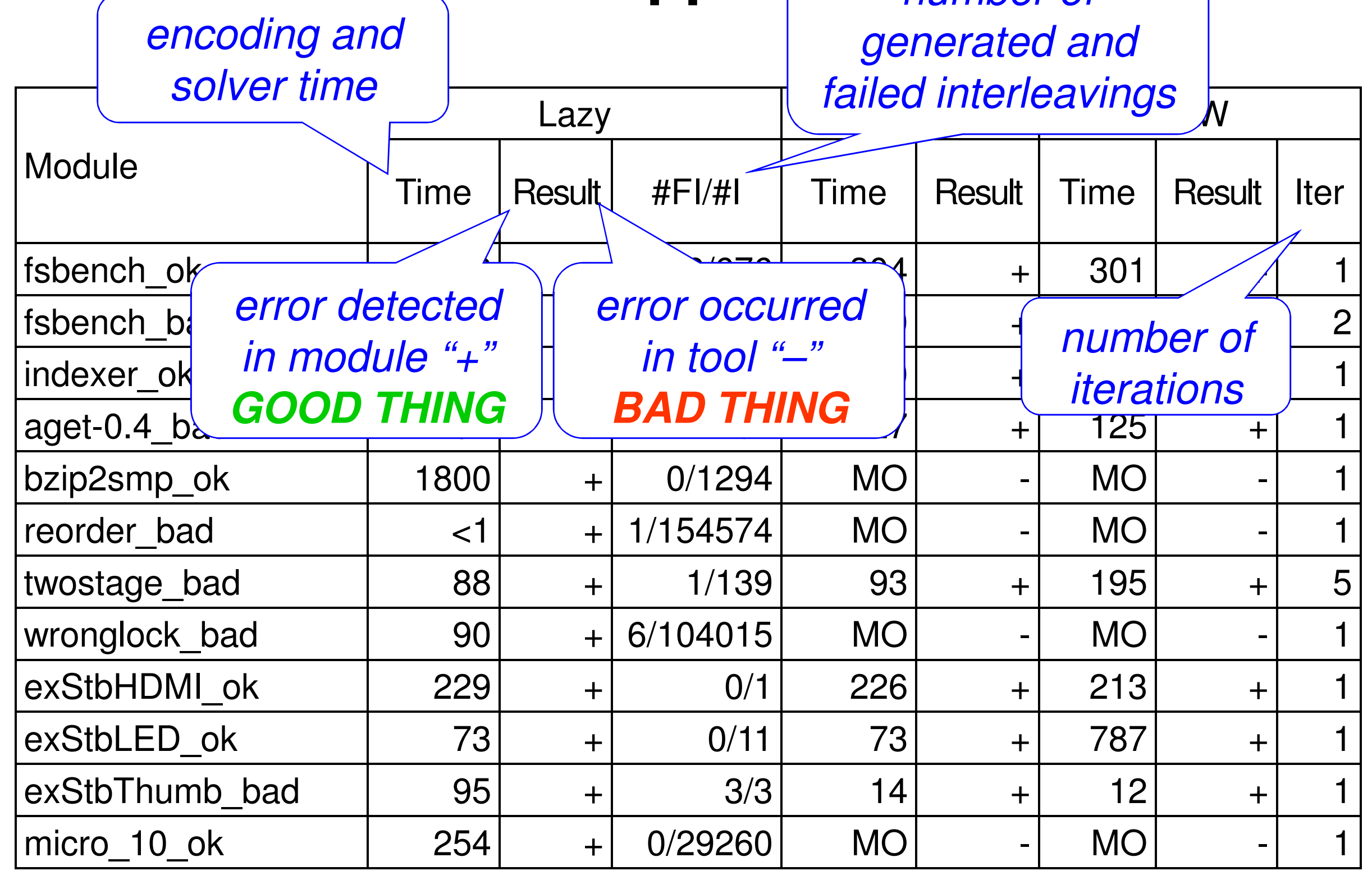




\section{Comparison of the approaches (1)}

\begin{tabular}{|c|c|c|c|c|c|c|c|c|}
\hline \multirow{2}{*}{$\begin{array}{c}\text { lazy encoding often } \\
\text { more efficient than } \\
\text { schedule recording } \\
\text { and UW }\end{array}$} & \multicolumn{3}{|c|}{ Lazy } & \multicolumn{2}{|c|}{ Schedule } & \multicolumn{3}{|c|}{ UW } \\
\hline & $g$ he & $\begin{array}{l}\text { Result } \\
-\end{array}$ & $\# \mathrm{Fl} / \# \mathrm{I}$ & Time & Result & Time & Result & Iter \\
\hline śperio 5 & 282 & + & $0 / 676$ & 304 & + & 301 & & \\
\hline fsbench_bad - & $<1$ & + & $729 / 729$ & 360 & + & 786 & & \\
\hline indexer_ok & $5 \overline{95}$ & $\mp$ & $-0 / \uparrow 7+6 \theta$ & --220 & $--_{+}^{-}$ & $\overline{2} 1 \overline{8}$ & + & 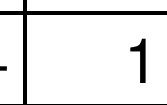 \\
\hline aget- 0 & 137 & -+ & $---4 / 1$ & --127 & $-\ldots+$ & .125 & + & - \\
\hline bzip2smp_ok & 1800 & + & $0 / 1294$ & MO & & MO & & \\
\hline reorder_bad & $<1$ & + & $1 / 154574$ & MO & - & MO & & \\
\hline twostage_bad & 88 & + & 139 & 93 & + & 195 & + & \\
\hline wronglock_bad - - & 90 & + & $6 / 104015$ & $\mathrm{MO}$ & & MO & - - = & \\
\hline exStbHDMI_ok & 229 & $\mp$ & $---0 / 4$ & --228 & + & $\overline{213}$ & + & 1 \\
\hline exStbLED_ok & 73 & + & $0 / 11$ & 73 & + & 787 & + & 1 \\
\hline exStbThumb_k & -95 & - \pm & $--3 / 3$ & --14 & - & 12 & - & \\
\hline micro_10_ok & 254 & + & $0 / 29260$ & MO & & MO & & \\
\hline
\end{tabular}




\section{Comparison of the approaches (2)}

\begin{tabular}{|c|c|c|c|c|c|c|c|c|}
\hline \multirow{3}{*}{\multicolumn{2}{|c|}{$\mid \begin{array}{c}\text { lazy encoding often more } \\
\text { efficient than schedule } \\
\text { recording and UW, but } \\
\text { not always }\end{array}$}} & \multicolumn{2}{|l|}{ azy } & \multicolumn{2}{|c|}{ Schedule } & \multicolumn{3}{|c|}{ UW } \\
\hline & & sult & \#Fl/\#I & Time & Result & Time & Result & Iter \\
\hline & & + & $0 / 676$ & 304 & + & 301 & + & 1 \\
\hline fsbench__ & $\leq 1$. & -+ & $729 / 729$ & --360 & $---t$ & -786 & + & 2 \\
\hline indexer_ok & 595 & + & $0 / 17160$ & 220 & + & 218 & $--x$ & 1 \\
\hline aget-0.4_bad - & 137 & + & $1 / 1$ & 127 & + & 125 & --7 & 1 \\
\hline bzip2smp_ok & $1 \overline{800}$ & + & $-0 /+294$ & --1 TOF & & $\mathrm{MO}$ & & 1 \\
\hline reorder_bad & $<1$ & + & \begin{tabular}{|l|}
$1 / 154574$ \\
\end{tabular} & $\mathrm{MO}$ & 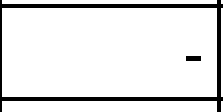 & $\mathrm{MO}$ & & t \\
\hline twostage_bad & 88 & + & $1 / 139$ & 93 & + & 195 & + & 5 \\
\hline wronglock_bad & -90 & + & $6 / 404015$ & $--A Q$ & ---- & $-\mathrm{MQ}$ & & 1 \\
\hline exStbHDMI_oK_- & 229 & + & $0 / 1$ & 226 & + & 213 & -+ & 1 \\
\hline exStbLED_ok & $\overline{3}$ & & $0<T 1$ & $=-73$ & 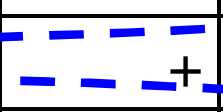 & -787 & + & 1 \\
\hline exStbThumb_bad & 95 & + & $3 / 3$ & 14 & + & 12 & \pm & 1 \\
\hline micro_10_ok & $\overline{254}$ & + & $0 / 29260$ & $-\mathrm{M} \mathrm{O}^{-1}$ & & $\overline{\mathrm{MO}}$ & & 1 \\
\hline
\end{tabular}




\begin{tabular}{|c|c|c|c|c|c|c|c|c|}
\hline \multicolumn{2}{|c|}{$\begin{array}{c}\text { lazy encoding is } \\
\text { extremely fast for } \\
\text { satisfiable instances }\end{array}$} & \multicolumn{7}{|c|}{ the approaches (3) } \\
\hline Módule & & Result & \#FI/\#I & Time & Result & Time & Result & Iter \\
\hline fsbench_ok & $2 \underline{2} 2$ & & $-0 / 676$ & -304 & & 301 & + & 4 \\
\hline fsbench_bad & $<1$ & + & $729 / 729$ & 360 & + & 786 & \pm & 2 \\
\hline indexer_ok & $\overline{595}$ & + & 0717160 & -220 & $\bar{\mp}$ & $\overline{218}$ & + & 1 \\
\hline aget-0.4_bad & 137 & + & $1 / 1$ & 127 & + & 125 & + & 1 \\
\hline bzip2smp_ok & 1800 & \pm & $-0 \leq 1294$ & $-M O$ & & $\mathrm{MO}$ & & 1 \\
\hline reorder_bad & $<1$ & + & $1 / 154574$ & MO & - & M̄̄ & $\therefore-5$ & 1 \\
\hline twostage_bad I & 88 & + & $1 / 139$ & 93 & + & 195 & + & 5 \\
\hline wronglock_bad & 90 & + & $6 / 104015$ & MO & & MO. & 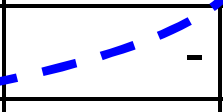 & 1 \\
\hline exStbHDMI_ok & $22 \overline{9}$ & + & $---0+1$ & --226 & $---\overline{+}$ & 213 & + & 1 \\
\hline exStbLED_ok & 73 & + & $0 / 11$ & 73 & + & 787 & + & 1 \\
\hline exStbThumb_bad & 95 & + & $3 / 3$ & 14 & + & 12 & + & -1 \\
\hline micro_10_ok & 254 & + & $0 / 29260$ & $\mathrm{MO}$ & 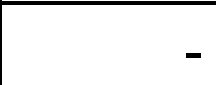 & $\mathrm{MO}$ & - & 1 \\
\hline
\end{tabular}




\section{Comparison to CHESS [Musuvathi and Qadeer]}

- CHESS (v0.1.30626.0) is a concurrency testing tool for C\# programs; also works for $\mathrm{C} / \mathrm{C}++$ (Windows $\mathrm{API})$.

- implements iterative context-bounding

- requires unit tests that it repeatedly executes in a loop, exploring a different interleaving on each iteration

- performs state hashing based on a happens-before graph

- avoids exploring the same state repeatedly

- Goal: compare efficiency of the approaches

- on identical verification problems taken from standard benchmark suites of multi-threaded software 


\begin{tabular}{l} 
CHESS is effective for $\begin{array}{l}\text { SS [Musuvathi and Qadeer] } \\
\text { programs where there are a } \\
\text { small number of threads }\end{array}$ \\
\cline { 2 - 7 } \\
\hline
\end{tabular}


CHESS is effective for programs where there are a small number of threads, but it does not scale that well and consistently runs out of time when we increase the number of threads

\begin{tabular}{|c|c|c|c|c|c|c|c|}
\hline \multirow{3}{*}{\multicolumn{3}{|c|}{$\begin{array}{l}\text { at well and consistently runs } \\
\text { it of time when we increase } \\
\text { e number of threads }\end{array}$}} & & \multicolumn{2}{|c|}{ CHESS } & \multicolumn{2}{|c|}{ Lazy } \\
\hline & & & & Time & Tests & Time & $\# \mathrm{Fl} / \# \mid$ \\
\hline & & & & & 130000 & $<1$ & $1 / 82$ \\
\hline reorder_5_bad $(4,1)$ _- & $-5 \mid$ & 5 & $\overline{6}$ & TO & 429000 & $<1$ & $=-1+277$ \\
\hline reorder_6_bad $(5,1)$ & 6 & 6 & 7 & TO & 396000 & $<1$ & $1 / 853$ \\
\hline reorder_6_bad $(5,4)$ & 6 & 6 & 8 & TO & 371000 & $<1$ & $1 / 2810$ \\
\hline reorder_6_bad $(5,1)$ & -6 & 6. & -9 & TO & 367000 & $-\leq 1$ & $- - 1 7 8 \longdiv { 1 2 4 }$ \\
\hline twostage_4_bad $(3,1)$ & 4 & 4 & 4 & 215 & -27000 & 2 & $1 / 42$ \\
\hline twostage_5_bad $(4,4)^{--}$ & 5 & 5 & 4 & TO & 384000 & 2 & $\overline{1} / 44$ \\
\hline twostage_6_bad $(5,1)-$ & $-\underline{6}$ & 6 & 4 & TO & 366000 & 2 & $-1 / 45$ \\
\hline wronglock_4_bad $(1,3)$ & 4 & 4 & 8 & 21 & 3000 & 5 & $2 / 489$ \\
\hline wronglock_5_bad $(1,4)$ & 5 & 5 & 8 & 72 & -93000 & 10 & $3 / 2869$ \\
\hline wronglock_6_badk $(I, 5)$ & 6 & 6 & 8 & TO & 356000 & 18 & $4 / 12 \overline{10} \overline{6}$ \\
\hline micro_2_ok (100) & 2 & 1 & 2 & 3101 & $\overline{3} \overline{5} \overline{8} \overline{5}$ & $\leq 1$ & $0 / 4$ \\
\hline micro_2_ok $(100)<$ & 2 & 1 & 17 & TO & 40000 & 1095 & $0 / 13 \overline{10} 7 \overline{2}$ \\
\hline
\end{tabular}

[Musuvathi and Qadeer]

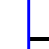




\section{Results}

- lazy, schedule recording, and UW algorithms

- lazy: check constraints lazily is fast for satisfiable instances and to a lesser extent even for safe programs

$\Rightarrow$ it has not been described or evaluated in the literature

- schedule recording: the number of threads and context switches can grow quickly (and easily "blow-up" the model checker)

$\Rightarrow$ combines symbolic with explicit state space exploration

- UW: memory overhead and slowdowns to extract the unsat core

$\Rightarrow$ it has not been used for BMC of multi-threaded software

\section{Future Work}

- fault localization in multi-threaded C programs

- verify real-time software using SMT techniques

- interpolants to prove non-interference of context switches 